\title{
Determinanten der kommunalen Steuerpolitik
}

\author{
Christian Person
}

Angenommen: 1. März 2021 / Online publiziert: 29. März 2021

(C) Der/die Autor(en) 2021

Zusammenfassung Die eigenverantwortliche Festsetzung der kommunalen Steuersätze stellt ein wichtiges Element der verfassungsrechtlich verbrieften Finanzautonomie kommunaler Gebietskörperschaften dar. Diese Handlungsspielräume werden von den Kommunen unterschiedlich wahrgenommen. Folglich bestehen zwischen den Kommunen erhebliche Divergenzen in der Ausgestaltung ihrer Steuerpolitik. Daher stellt sich die Frage, wie sich diese Diskrepanzen erklären lassen. Sind diese auf sozioökonomische, budgetäre oder parteipolitische Einflussfaktoren zurückzuführen? Zur Klärung dieser Frage wird im Rahmen des Beitrags die kommunale Realsteuerpolitik in sechs Bundesländern zwischen 2004 und 2013 analysiert. Dabei stellen sich vor allem sozioökonomische und budgetäre Einflussfaktoren als erklärungskräftig heraus, wohingegen Parteieneffekte nur vereinzelt festzustellen sind. Dies verdeutlicht, dass der Handlungsspielraum von Parteien auf lokaler Ebene durch den wachsenden fiskalischen Problemdruck zunehmend eingeengt wird. Lediglich bei den Grundsteuern scheinen begrenzte Gestaltungsspielräume zu bestehen, die sich teilweise in parteipolitisch bedingten Differenzen manifestieren. Durch die Identifikation relevanter Determinanten der kommunalen Steuerpolitik leistet der Aufsatz somit einen Beitrag zu einem besseren Verständnis lokaler Haushaltspolitik.

Schlüsselwörter Kommunalpolitik · Kommunalfinanzen · Gewerbesteuer · Grundsteuer · Hebesatzpolitik · Determinanten · Policy-Analyse

Dr. C. Person $(\bowtie)$

Institut für Politikwissenschaft, Arbeitsbereich Öffentliche Verwaltung, Public Policy, Technische Universität Darmstadt, Dolivostraße 15, 64293 Darmstadt, Deutschland

E-Mail: christian.person@tu-darmstadt.de 


\section{Determinants of local tax policy}

Abstract The autonomous choice of local tax rates is an important element of local fiscal autonomy, guaranteed by the German constitution. As municipalities use this option differently, there are large discrepancies in the design of local tax policy. This raises the question how these differences can be explained. To answer the question, the article analyses the determinants of local tax policy in six German Länder between 2004 and 2013. The analysis reveals that economic and budgetary conditions are main drivers of local tax policy. Partisan effects can be identified only sporadically. Thus, the ability of parties to influence local tax policy according to their preferences is increasingly restrained by growing fiscal problem pressure. Only with regard to local property taxes, they seem to have some room for manoeuvre. Hence, by identifying important determinants of local tax policy, the article contributes to a better understanding of local budget politics.

Keywords Local politics $\cdot$ Local public finance $\cdot$ Business tax $\cdot$ Property tax Local tax policy $\cdot$ Determinants $\cdot$ Policy analysis

\section{Einleitung}

Das Grundgesetz garantiert den Kommunen umfangreiche Selbstverwaltungsrechte. Diese umfassen auch die Grundlagen der finanziellen Eigenverantwortung. Ein Baustein dieser Finanzautonomie ist die Festlegung der Hebesätze bei den Realsteuern (Grundsteuer A und B sowie Gewerbesteuer). Durch die Festsetzung der Steuersätze können Kommunen ihre Einnahmen an ihre Finanzbedarfe anpassen, um ein hochwertiges, den Präferenzen der Bevölkerung entsprechendes Leistungsangebot bereitzustellen. Gleichzeitig beeinflusst dies ihre Anziehungskraft als Unternehmensstandort und Wohnsitz (Schwarting 2019, S. 91-92).

Die Ausgestaltung der Steuerpolitik divergiert zwischen den Kommunen erheblich wie die Realsteuerstatistik 2019 verdeutlicht. So begnügt sich bei der Gewerbesteuer die Stadt Zossen mit dem Mindesthebesatz von 200 Punkten, wohingegen Wettlingen einen drei Mal so hohen Hebesatz erhebt. Noch deutlicher sind die Unterschiede bei den Grundsteuern. Während 16 Kommunen bei der Grundsteuer A, 13 bei der Grundsteuer B, einen Hebesatz von 0 festlegen, liegt dieser in acht Kommunen bei über 1000. Auch zwischen den Bundesländern kann man große Unterschiede beobachten. Sowohl bei der Gewerbesteuer als auch der Grundsteuer B weisen insbesondere Hessen, Nordrhein-Westfalen und das Saarland hohe Hebesätze auf. Dies überrascht kaum, da diese Länder seit Jahren mit lokalen Haushaltskrisen konfrontiert sind, die sie jüngst mittels Entschuldungsprogrammen adressierten (Person und Geißler 2020). Seither unterliegen die Kommunen massivem Druck, ihre Hebesätze zum Zwecke der Haushaltskonsolidierung anzuheben.

Überdies lässt sich seit Jahrzehnten ein sukzessiver Anstieg der Hebesätze konstatieren. Seit Gründung der BRD hat sich der bundesweite Durchschnittshebesatz der Grundsteuern mehr als verdoppelt und stieg bei der Grundsteuer A von 150 auf 342 Punkte, bei der Grundsteuer B von 208 auf 475. Im Fall der Gewerbesteu- 
er fiel die Dynamik schwächer aus. Dennoch nahm der Hebesatz spürbar von 264 auf 403 Punkte zu (Destatis o.J.). Insbesondere in der jüngeren Vergangenheit ließen sich in zahlreichen Kommunen Steuererhöhungen beobachten, die darauf hindeuten, dass diese in einer „Hebesatzspirale“, einem „Teufelskreis aus immer höheren Steuersätzen und immer weniger Investitionen“ (Wagschal et al. 2016, S. 15) gefangen sind.

Die Varianz der Hebesätze im Querschnitt und die dynamische Entwicklung im Längsschnitt werfen die Frage auf, was diese Unterschiede begründet. Um dies $\mathrm{zu}$ prüfen, widmet sich der Beitrag folgender Forschungsfrage: Welche Faktoren bestimmen die Ausgestaltung der Realsteuerhebesatzpolitik und unterscheiden sich diese zwischen einzelnen Realsteuerarten? Zur Beantwortung der Fragestellung wird unter Verwendung von OLS-Regressionsverfahren für Paneldaten die Hebesatzpolitik in sechs Bundesländern zwischen 2004 und 2013 untersucht. Dabei werden neben sozioökonomischen und budgetären Prädiktoren auch politische Einflussfaktoren berücksichtigt.

Der Beitrag gliedert sich wie folgt: Kapitel zwei referiert den Forschungsstand zu den Determinanten der Steuerpolitik, insbesondere der Realsteuerhebesatzpolitik. Darauf aufbauend werden im dritten Kapitel das theoretische Modell und die Hypothesen erläutert. Im vierten Kapitel wird das Forschungsdesign vorgestellt. Die empirischen Befunde präsentiert Kapitel fünf. Das letzte Kapitel zieht ein Fazit.

\section{Forschungsstand}

Die Ausgestaltung der Steuerpolitik als Teilgebiet der öffentlichen Finanzen stellt seit jeher einen relevanten Analysegegenstand der ökonomischen und der politikwissenschaftlichen Forschung, speziell der vergleichenden Policy-Analyse, dar. Insbesondere unterschiedliche Niveaus der Besteuerung und Strukturen der Steuersysteme zwischen Nationalstaaten sowie deren Bestimmungsfaktoren fanden viel Beachtung in der Forschung (eine Übersicht bietet Wagschal 2006; Wagschal 2015, S. $506 \mathrm{ff}$.). Unter Rückgriff auf das theoretische Instrumentarium der vergleichenden Staatstätigkeitsforschung lassen sich auf Basis internationaler Vergleiche die Determinanten der Steuerpolitik in sechs Gruppen unterteilen (Wagschal 2005, S. 25 ff.). Im Einklang mit der sozioökonomischen Schule stellt Staatstätigkeit eine Reaktion auf gesellschaftliche und wirtschaftliche Herausforderungen dar (Wilensky 1975). Demnach zwingt sozioökonomischer Problemdruck (hohe Arbeitslosigkeit, viele Transferempfänger, schwache wirtschaftliche Entwicklung) Regierungen dazu, ihre fiskalpolitischen Instrumente anzupassen. Die Richtung dieses Wandels ist jedoch umstritten. Einerseits wird argumentiert, dass budgetärer Druck zu Steuererhöhungen führt, um den Haushalt wieder ausgleichen zu können. Andererseits wird betont, Staaten könnten als Reaktion auch Abgaben senken, um Anreize zur Unternehmensansiedlung zu schaffen und die wirtschaftliche Entwicklung zu fördern (von Kulessa und Wenzelburger 2015, S. 307). Neben dieser funktionalistischen Perspektive wird auch die Rolle politischer Akteure näher beleuchtet. In Übereinstimmung mit der Parteiendifferenztheorie (Hibbs 1977; Tufte 1978) wurden parteipolitisch geprägte Einflüsse auf die Steuerpolitik nachgewiesen, sowohl mit Blick auf die Höhe und 
Progressivität der Besteuerung als auch die Struktur des Steuersystems, wobei Linksparteien mit höheren Abgabequoten in Verbindung gebracht werden. Demgegenüber betonen machtressourcenbasierte und pluralistische Theorieansätze (Korpi 1983; Olson 1982) die Rolle von organisierten Interessen, korporatistischer Strukturen und Verteilungskoalitionen für die Staatstätigkeit. In Bezug auf die Steuerpolitik wird angenommen, dass die Berücksichtigung vielfältiger Partikularinteressen zu einem höheren Finanzbedarf und einer umfassenderen Erhebung von Abgaben führt. Auch die institutionelle Struktur, insbesondere die Existenz gegenmajoritärer Institutionen und die Zahl der Vetospieler (Tsebelis 2002), beeinflusst die Steuerpolitik. Dabei wirken Vetospieler tendenziell begrenzend auf die Höhe der Besteuerung und die Wahrscheinlichkeit von Steuerreformen. Vielfach diskutiert wird zudem der Einfluss der zunehmenden Integration der Nationalstaaten in die Weltwirtschaft im Zuge der Globalisierung und der sich daraus ergebende internationale Steuerwettbewerb. Das in diesem Zusammenhang befürchtete sukzessive Absinken der Steuersätze (race to the bottom) fand empirisch jedoch keine klare Bestätigung. Dies ist aus Sicht von Vertretern der Politik-Erblast-Theorie (Rose und Karran 1987; Rose und Davies 1994) wenig überraschend. Ihrer Ansicht nach ist der Handlungsspielraum von Regierungen durch historische Erblasten stark eingeschränkt, d.h. die Staatstätigkeit früherer Zeiten reduziert die Handlungsspielräume der Regierungen im Hier und Jetzt. Steuersysteme zeichnen sich durch eine hohe Trägheit und ein großes Beharrungsvermögen aus, so dass allenfalls inkrementelle Fortentwicklungen möglich sind (Wagschal 2005; Wagschal 2006).

Allerdings konzentriert sich die Forschung zu den Determinanten der Steuerpolitik primär auf die Ebene der Nationalstaaten, wohingegen die kommunale Steuerpolitik weniger Beachtung findet. Deutschland bildet hierbei keine Ausnahme. Es bleibt somit offen, inwieweit die zuvor skizzierten Erklärungsansätze auch auf lokaler Ebene Anwendung finden. Um dies zu prüfen, wird im Folgenden der Forschungsstand zu den Bestimmungsfaktoren der Realsteuern in Deutschland dargelegt. Dabei fällt auf, dass die Determinanten der Gewerbesteuerhebesatzpolitik insbesondere im Rahmen ökonomischer Studien analysiert werden, welche sich auf sozioökonomische und budgetäre Prädiktoren fokussieren. In diesem Kontext wurden fiskalische Kapazitäten als wichtiger Einflussfaktor identifiziert. So findet Büttner (1999, 2000) einen negativen Zusammenhang zwischen dem kommunalen Einkommensteueranteil, den Schlüsselzuweisungen oder dem Grundbetrag der Gewerbesteuer und dem Hebesatz. Auch Hauptmeier et al. (2012) weisen negative Effekte von Finanzzuweisungen nach, während dies Boettcher (2013b) und Person (2020) für den Gewerbesteuergrundbetrag gelingt. Einen weiteren, relevanten Einflussfaktor stellen kommunale Fixkosten dar. Dies sind Ausgabearten, die eine Kommune selbst kaum beeinflussen kann. Sie sind primär durch exogene Entscheidungen und Entwicklungen bestimmt. Diesbezüglich identifizieren Büttner (2000), Boettcher (2013b) sowie Fuest und Riphahn (2001) positive Effekte von Sozial- und Zinsausgaben auf das Hebesatzniveau. Auch Ausgaben für die Kreisumlage wird eine hebesatzsteigernde Wirkung attestiert (Büttner 2001). Abschließend ist bei diesen budgetären Faktoren die allgemeine Haushaltslage zu nennen, die ebenfalls die Hebesatzpolitik beeinflusst. So finden Koh et al. (2013) einen positiven Zusammenhang zwischen Budgetdefiziten und Hebesatzniveau. Person (2020) zeigt, dass die Verschuldung 
einen positiven Effekt auf den Gewerbesteuerhebesatz entfaltet. Dies beobachten auch Seitz (1995) und Fehrenz (2019).

Neben budgetären Einflussfaktoren spielen räumliche Lageparameter sowie sozioökonomische und -demografische Faktoren eine wichtige Rolle für die kommunale Steuerpolitik. Sowohl für den Urbanisierungsgrad (Kunz 2000; Koh et al. 2013) als auch die Standortattraktivität einer Kommune (Boettcher 2013b) wurden positive Effekte auf das Hebesatzniveau nachgewiesen. Der Einfluss der räumlichen Lage ist ebenfalls nicht zu vernachlässigen. Nach Kunz (2000) wirkt sich die Lage in einem Agglomerationsraum hebesatzsteigernd aus, wohingegen Koh et al. (2013) einen negativen Effekt für ländliche Gebiete finden. Überdies wurde verschiedentlich ein positiver Zusammenhang zwischen Gemeindegröße und Hebesatzniveau identifiziert (Büttner 2001; Hauptmeier et al. 2012). Daneben spielt auch die Bevölkerungsstruktur eine Rolle. Büttner (2001) findet einen negativen Effekt für den Anteil der unter 15-, 25- und 65-Jährigen an der Gesamtbevölkerung im Vergleich zur Referenzgruppe (Personen über 65 Jahre). Er erklärt dies mit einer höheren Nachfrage der Senioren nach öffentlichen Leistungen. Demgegenüber beobachtet Beck (2019) sowohl für den Seniorenanteil als auch den Anteil der Personen unter 18 sowie zwischen 18 und 30 Jahren eine hebesatzsteigernde Wirkung. Auch Koh et al. (2013) konstatieren einen positiven Effekt der Seniorenquote.

Ambivalente Befunde zeigen sich mit Blick auf den sozioökonomischen Problemdruck, der auf nationalstaatlicher Ebene ein wichtiger Treiber der Steuerpolitik darstellt. Nach Kunz (2000) wirken sich die Arbeitslosen- und die Sozialhilfequote hebesatzerhöhend aus. Auch Koh et al. (2013) und Fehrenz (2019) finden positive Effekte der Arbeitslosenquote. Demgegenüber beobachten Büttner und Franz (2003) sowie Hauptmeier et al. (2012) einen hebesatzsenkenden Einfluss.

Analog zur nationalen Ebene wird auch mit Blick auf die Kommunen die Rolle des Steuerwettbewerbs diskutiert. Dabei wird der interkommunale Steuerwettbewerb als relevanter Einflussfaktor für die gemeindliche Steuersatzwahl identifiziert. Demnach orientieren sich Kommunen bei der Wahl ihres Gewerbesteuerhebesatzes an ihren Nachbargemeinden (Boettcher 2013b; Büttner 2000; Hauptmeier et al. 2012) oder Gemeinden mit ähnlicher Wirtschaftsstruktur (Seitz 1995), ohne dass dies jedoch wie oftmals befürchtet in einer Abwärtsspirale der Steuersätze kulminiert. Zudem zeichnet sich die Hebesatzpolitik im Einklang mit den Erwartungen der Politik-Erblast-Theorie durch eine hohe Pfadabhängigkeit und lediglich inkrementellen Änderungen aus, da Vorjahreswerte eine wichtige Orientierungsgröße bei der Hebesatzwahl darstellen (Büttner 2000; Büttner und Franz 2003).

Im Gegensatz zu sozioökonomischen und budgetären Prädiktoren stand der Einfluss (partei-)politischer Faktoren kaum im Fokus des Erkenntnisinteresses (Person 2020, S. 64). Dies wird darauf zurückgeführt, dass Lokalpolitik durch eine unpolitische Selbstverwaltungstradition und eine starke Konsensorientierung geprägt sei. Parteiendifferenztheoretische Ansätze wären daher nur begrenzt anwendbar (Gabriel et al. 1994, S. 160). Zudem bedinge die kommunale Finanznot einen wachsenden Einfluss der Aufsichtsbehörden, der Parteieneffekte überlagere (Boettcher 2013b; Timm-Arnold 2011, S. 272). Folgerichtig liefern Studien zur Parteiendifferenz auf lokaler Ebene nur schwache Belege für eine inhaltliche Parteipolitisierung (Bogumil und Holtkamp 2006, S. 137; Person 2020, S. 65). 
Diese Einschätzung bestätigt sich für die Gewerbesteuerpolitik, die sich bezüglich parteipolitischer Einflussfaktoren durch ambivalente Befunde auszeichnet. TimmArnold (2011, S. 284) zeigt in einer Analyse der Haushaltspolitik von fünf Ruhrgebietsstädten, dass linke Ratsmehrheiten eher Steuern erhöhen. Konservative Parteien versuchen, dies zu vermeiden. Auch Kunz (2000) belegt in einer Analyse der Budgetpolitik von 87 Großstädten, dass SPD-Mehrheiten zu höheren Hebesätzen führen. Bogumil und Holtkamp (2006, S. 136-137) zweifeln jedoch die Generalisierbarkeit der Befunde an. Erstens berücksichtigt die Studie nur kreisfreie Städte. Zweitens beschränkt sie sich auf die 1980er-Jahre, in denen Kommunalhaushalte selten dramatische Schieflagen aufwiesen, welche die Handlungsmöglichkeiten der Parteien zunehmend einschränken. Demgegenüber sind die Befunde bei Fehrenz (2019) weniger eindeutig. Zwar beobachtet sie eine positive Beziehung zwischen dem SPDSitzanteil im Stadtrat und dem Hebesatzniveau. Dieser ist jedoch nur teilweise signifikant. Zudem stellen sich der CDU-Sitzanteil und die Parteizugehörigkeit des Bürgermeisters als insignifikant heraus. Boettcher (2013b) findet ebenfalls keine Belege für parteipolitische Einflüsse. Auch Person (2020) identifiziert keine generellen Parteieneffekte, unabhängig davon, ob man die Mehrheitsverhältnisse im Rat oder die Parteizugehörigkeit des Bürgermeisters betrachtet.

Während zahlreiche Studien zur Gewerbesteuerpolitik vorliegen, wurden die Grundsteuern stiefmütterlich behandelt. Nur vier Arbeiten wurden identifiziert, welche die Determinanten der Grundsteuer B analysieren, mit Blick auf die Grundsteuer A gar nur eine. Analog zur Gewerbesteuer besteht für beide Grundsteuern ein negativer Zusammenhang zwischen Einnahmekapazitäten und Hebesatzniveau. Auch der interkommunale Steuerwettbewerb beeinflusst die Wahl des Hebesatzes, da das Hebesatzniveau der Nachbargemeinden einen positiven Effekt auf den eigenen Hebesatz entfaltet. Widersprüchlicher sind die Befunde zur Bevölkerungsstruktur. Während sich bei der Grundsteuer A ein positiver Effekt des Anteils an Personen unter 18, zwischen 18 und 30 sowie über 65 Jahren zeigt, gilt dies im Falle der Grundsteuer B nur für Personen zwischen 18 und 30 Jahren. Die anderen Gruppen wirken sich negativ auf das Hebesatzniveau aus (Beck 2019). Zu einem ähnlichen Ergebnis in Bezug auf Kinder kommt auch Kunz (2000). Ebenfalls konträre Befunde zeigen sich für die Standortattraktivität. Während die Erreichbarkeit zentraler Infrastrukturen, die Beschäftigtenquote, der Pendlersaldo und die Einwohnerzahl bei der Grundsteuer B einen positiven Effekt auf den Hebesatz entfaltet, wirken diese Faktoren bei der Grundsteuer A negativ (Beck 2019).

Überdies stellt sich für die Grundsteuer B sozioökonomischer Problemdruck als erklärungskräftig dar: Je höher die Arbeitslosen- und Sozialhilfequote, desto höher die Hebesätze (Kunz 1998; Fehrenz 2019). Das gleiche gilt für die kommunale Verschuldung (Fehrenz 2019). Widersprüchlicher sind die Befunde zur Standortattraktivität und zur Lage im Raum. Einerseits berichtet Kunz (1998), dass sich die Lage in einem Agglomerationsraum positiv auf das Hebesatzniveau auswirkt. Andererseits findet er einen negativen Effekt der Arbeitsmarktzentralität. Letzteres steht im Widerspruch zu Beck (2019) und Fehrenz (2019), die einen positiven Effekt der Bevölkerungsdichte, einem weiteren Indikator für die Standortattraktivität, konstatieren. Auch im Hinblick auf parteipolitische Einflussfaktoren sind die Befunde uneinheitlich. Während Kunz (2000) für die Grundsteuer B keine Parteieneffekte 
findet, ist bei Fehrenz (2019) das Gegenteil der Fall. Nach ihrer Analyse hat der CDU-Sitzanteil im Stadtrat einen negativen Effekt auf das Hebesatzniveau. Demgegenüber zeigt sich für den SPD-Sitzanteil ein positiver Effekt, der jedoch teilweise insignifikant ist. Keine signifikanten Parteieneffekte sind für Bürgermeister festzustellen.

Es bleibt festzuhalten, dass bis dato die Gewerbesteuerpolitik im Fokus des Erkenntnisinteresses stand. Studien zu den Determinanten der Grundsteuer sind rar. Somit besteht kaum gesichertes Wissen, ob Hebesatzentscheidungen bei allen Realsteuern von den gleichen Faktoren abhängen oder realsteuer-spezifische Einflüsse existieren. Ein weiteres Defizit stellt die Dominanz ökonomischer Studien dar, welche eine Fokussierung auf sozioökonomische und budgetäre Prädiktoren bedingt. Die Rolle politischer Einflussfaktoren wurde vernachlässigt. Überdies sind einige Studien älterer Natur und nutzen oft die 1980er/1990er als Untersuchungszeitraum. Allerdings haben sich in den letzten zwei Dekaden die Rahmenbedingungen der Kommunalpolitik durch Haushaltskrisen und Kommunalverfassungsreformen stark gewandelt. Daher wäre die Nutzung aktuellerer Beobachtungszeiträume wünschenswert, um zu prüfen, wie die Hebesatzpolitik unter diesen neuen Bedingungen funktioniert. Des Weiteren sind einige Studien, v.a. zur Grundsteuer, reine Querschnittsanalysen. Diese verwenden nur ein Untersuchungsjahr (Beck 2019; Fehrenz 2019) oder den Mittelwert bzw. die Änderung einzelner Variablen über mehrjährige Betrachtungszeiträume (Kunz 1998, 2000). Im ersten Fall bleibt fraglich, ob die Befunde durch besondere, nur in diesem Untersuchungsjahr vorliegende Bedingungen beeinflusst und somit generalisierbar sind. Im zweiten Fall wird Analysepotential verschenkt, da das statistische Verfahren die zeitliche Struktur der Daten nicht berücksichtigt. Auch die Fallauswahl ist problematisch, da diese häufig stark eingeschränkt ist. Entweder werden Kommunen mit spezifischen Charakteristika ausgewählt, z.B. kreisfreie Städte (Kunz 1998, 2000), Großstädte (Fuest und Riphahn 2001), Städte mit über 50.000 Einwohnern (Fehrenz 2019), oder alle Kommunen eines Bundeslandes, wobei man sich meistens auf Baden-Württemberg oder Nordrhein-Westfalen beschränkt. Beide Ansätze werfen Fragen nach der Repräsentativität der Untersuchungseinheiten und der Generalisierbarkeit der Befunde auf. Die erste Herangehensweise ignoriert die kleinteilige Struktur der Gemeindeebene, da $85 \%$ der Kommunen weniger als 10.000 Einwohner aufweisen (Stand 2019). Der zweite Ansatz vernachlässigt unterschiedliche Rahmenbedingungen der Lokalpolitik, da zwischen den Ländern große Unterschiede bezüglich Gemeindestruktur und Kommunalverfassung bestehen.

Vor diesem Hintergrund trägt der Beitrag in mehrfacher Hinsicht zum Forschungsstand bei. Erstens wird durch die simultane Analyse der Determinanten aller Realsteuerarten ein besseres Verständnis der kommunalen Steuerpolitik erzielt, v. a. mit Blick auf Gemeinsamkeiten und Unterschiede zwischen den Realsteuern. Zweitens wird durch die Berücksichtigung politischer Erklärungsfaktoren der dominierende ökonomische Blick auf die lokale Steuerpolitik um eine politikwissenschaftliche Perspektive ergänzt. Drittens wird durch die Verwendung eines aktuelleren Untersuchungszeitraums den veränderten Rahmenbedingungen der kommunalen Steuerpolitik Rechnung getragen. Viertens wird durch die Berücksichtigung mehrerer Bundes- 
länder die Repräsentativität der Untersuchungseinheiten und die Generalisierbarkeit der Befunde erhöht.

\section{Theorie}

Nach Boettcher (2013a) kann man die Determinanten der Hebesatzentscheidung in drei Kategorien unterteilen. Das Hebesatzanspannungspotential erfasst die Fähigkeit einer Kommune, ihren Hebesatz anzuspannen, ohne negative Folgen für ihren Haushalt fürchten zu müssen. Der Hebesatzanspannungsbedarf signalisiert, inwieweit sie diesen aufgrund fiskalischer Erfordernisse anspannen muss, um ausreichende Einnahmen zu erzielen. Während beide Kategorien von funktionalistischen Wirkzusammenhängen ausgehen und aus politikwissenschaftlicher Perspektive ideell der Sozioökonomischen Schule der Staatstätigkeitsforschung nahestehen, betont die dritte Kategorie, der Hebesatzanspannungswille, die Rolle der Politik. Demnach wird die Hebesatzentscheidung auch von den politischen Präferenzen der Entscheidungsträger beeinflusst.

\subsection{Das Hebesatzanspannungspotential}

Das Hebesatzanspannungspotential hängt von zwei Faktoren ab: der Zahlungsfähigkeit und der Zahlungsbereitschaft der Steuerzahler. Erstere erfasst, welche Steuerlast für die Steuerzahler ökonomisch tragbar ist. Letztere gibt an, welche Steuerlast sie zu tragen bereit sind. Die Zahlungsfähigkeit der Steuerzahler richtet sich nach ihrer ökonomischen Leistungsfähigkeit. Üblicher Indikator ist das Bruttoinlandsprodukt oder die Bruttowertschöpfung. Allerdings liegen beide Kennziffern auf der Gemeindeebene nicht vor. Als Alternative verwenden Studien zur Gewerbesteuerpolitik den Grundbetrag der Gewerbesteuer. Dieser liefert ein Abbild der lokalen Steuerbasis und damit der Leistungsfähigkeit der Steuerpflichtigen. Dabei wird ein positiver Zusammenhang zwischen Leistungsfähigkeit und Hebesatzanspannung angenommen, da ertragsstarke Unternehmen höhere Steuerlasten besser verkraften als ertragsschwache. Je umfassender die wirtschaftliche Basis einer Kommune, desto eher zeigen sich negative Effekte der Besteuerung erst bei hohen Hebesätzen (Boettcher 2013a). Für die Grundsteuern existieren in der Literatur keine analogen Überlegungen. Vereinfachend wird ein ähnlicher Wirkzusammenhang unterstellt. Daraus ergibt sich folgende Hypothese:

$\mathbf{H}_{1}$ Je höher die Grundbeträge der Realsteuern, desto stärker können Kommunen ihre Realsteuerhebesätze anspannen.

Darüber hinaus hängt das Hebesatzanspannungspotential von der Zahlungsbereitschaft der Steuerzahler ab. Diese ist eine Funktion der Standortattraktivität. Die Standortattraktivität einer Kommune wird von zahlreichen Faktoren beeinflusst und richtet sich nicht nur nach der Abgabenbelastung (Grabow und Hollbach-Grömig 1994). Vielmehr ist entscheidend, welche Leistungen den Steuerpflichtigen im Austausch für ihre Steuerzahlungen bereitgestellt werden. Bieten Kommunen attraktive 
Leistungen, können sie höhere Abgaben verlangen. Aus Unternehmersicht wird die Standortattraktivität durch die Existenz von Agglomerationsvorteilen geprägt, da sie die Markterschließung vereinfachen. Hierzu zählen größere Zulieferer- und Absatzmärkte, niedrigere Transportkosten aufgrund der Nähe von Zulieferern und Abnehmern, ein ausdifferenziertes Arbeitskräfteangebot, positive technologische Externalitäten, z.B. Wissenstransfers zwischen Unternehmen eines Wirtschaftsclusters, eine lebendige Forschungslandschaft, eine ausgebaute Infrastruktur und eine gute Verkehrsanbindung (Eckey 2008; Krugman 1991; Berlemann und Tilgner 2007). Für Bürger dürfte die ökonomische Standortattraktivität bei Wohnsitzentscheidungen relevant sein, da sie mit einem breiten Angebot an hochwertigen, gut entlohnten Beschäftigungsmöglichkeiten und einem ausdifferenzierten öffentlichen Leistungsangebot einhergeht.

Folglich hängt die Standortattraktivität davon ab, wie viele Unternehmen sich vor Ort niederlassen. Daher wird als Indikator die Arbeitsplatzzentralität verwendet. Sie erfasst die Bedeutung einer Kommune als Arbeitsort und spiegelt ihre ökonomische Attraktivität. Die Arbeitsplatzzentralität wird als Pendlersaldo (Einpendler abzüglich Auspendler) pro 100 Einwohner im erwerbsfähigen Alter zwischen 15 und 65 Jahren operationalisiert (Boettcher 2013a). Ein alternativer Indikator, v. a. mit Blick auf das Markterschließungsmotiv, ist die Bevölkerungsdichte (Fehrenz 2019, S. 533). Eine höhere Bevölkerungsdichte bietet Unternehmen größere lokale Absatzmärkte und Faktorangebotsmärkte, z. B. im Hinblick auf das Arbeitskräfteangebot (Berlemann und Tilgner 2007). Dies führt zu folgenden Hypothesen:

$\mathbf{H}_{2}$ Je höher die Arbeitsplatzzentralität, desto stärker können Kommunen ihre Realsteuerhebesätze anspannen.

$\mathbf{H}_{3}$ Je höher die Bevölkerungsdichte, desto stärker können Kommunen ihre Realsteuerhebesätze anspannen.

Weiterhin argumentieren Büttner und Franz (2003), mit Blick auf die Gewerbesteuer, dass Hebesätze auch als Instrument zur Beeinflussung der Wettbewerbsposition fungieren. Demnach besitzen Kommunen im Falle hoher Arbeitslosigkeit Anreize, ihre Hebesätze zu senken, um ihre Standortattraktivität zu erhöhen. Die geringere Abgabenlast soll Unternehmen dazu bewegen, sich vor Ort anzusiedeln oder bestehende Kapazitäten auszubauen. Dadurch entstünden zusätzliche Arbeitsplätze, die Arbeitslosigkeit würde bekämpft. ${ }^{1}$ Daher wird folgende Hypothese getestet:

$\mathbf{H}_{4}$ Je höher die Arbeitslosigkeit, desto niedriger sind die Realsteuerhebesätze.

Ein weiterer Parameter der Hebesatzentscheidung dürfte die Gemeindegröße sein. Auf Basis der Optimalsteuertheorie und theoretischer Abhandlungen zum Steuer-

\footnotetext{
1 Diese Argumentationskette spiegelt Diskussionen wieder, wie sie in der international vergleichenden Forschung zur Unternehmensbesteuerung mit Blick auf die Rolle des internationalen Steuerwettbewerbs und des binnenwirtschaftlichen Problemdrucks geführt werden (vgl. von Kulessa und Wenzelburger 2015, S. $305 \mathrm{ff}$.$) .$
} 
wettbewerb wird angenommen, dass größere Gebietskörperschaften höhere Steuersätze festlegen können als kleinere, da sie über größere Anteile am gemeinsamen Kapitalmarkt verfügen. Dadurch fällt der Effekt einer Steuersatzänderung auf die Menge des vor Ort investierten Kapitals in größeren Gebietskörperschaften geringer aus. Folglich besteht eine positive Beziehung zwischen Gemeindegröße und Hebesatzanspannungspotential (Bucovetsky 1991; Wilson 1991; Büttner 2001). Daraus ergibt sich folgende Hypothese:

H5 Je größer eine Kommune, desto höher sind ihre Realsteuerhebesätze.

\subsection{Der Hebesatzanspannungsbedarf}

Neben dem Hebesatzanspannungspotential beeinflusst der Hebesatzanspannungsbedarf die Wahl des Hebesatzes. Hierbei stellt die Finanzsituation einer Kommune einen wichtigen Einflussfaktor dar. Weist sie eine hohe Finanzkraft auf, also umfangreiche allgemeine Deckungsmittel (Einnahmen aus Realsteuern, Gemeinschaftssteueranteilen sowie ungebundenen Zuweisungen), schmälert dies ihren Hebesatzanspannungsbedarf. Sie ist nicht gezwungen, hohe Hebesätze festzusetzen, um eine schwache Finanzausstattung zu kompensieren, sondern kann auf üppige Finanzmittel zurückgreifen (Boettcher 2013a; Büttner 2000, S. 66; Kunz 1998, S. 172). Dies führt zu folgender Hypothese:

H6 Je höher die Finanzkraft einer Kommune, desto niedriger sind ihre Realsteuerhebesätze.

Konzentriert man sich auf die Realsteuern als wichtigste, autonom gestaltbare Einnahmequellen, ist von einem negativen Effekt des Grundbetrages auf den Hebesatz auszugehen. Kommunen, die bei einzelnen Realsteuern hohe Grundbeträge aufweisen, können ihre Steuerpflichtigen vor hohen Hebesätzen schützen, da sie aufgrund der breiten Steuerbasis selbst bei niedrigen Hebesätzen hohe Erträge generieren. Demgegenüber sehen sich Kommunen mit einer geringeren Steuerbasis zu einer Anspannung der Hebesätze genötigt, um äquivalente Erträge zu erzielen (Boettcher 2013a; Büttner und Franz 2003; Postlep 1985, S. 181ff.). Daraus ergibt sich folgende Hypothese:

$\mathbf{H}_{7} \quad$ Je höher die Grundbeträge einzelner Realsteuern, desto geringer der Realsteuerhebesatz.

Bei einem gegebenen Ausgabebedarf stehen Kommunen vor der Wahl, welche Realsteuer zur Finanzierung herangezogen werden soll. Dabei ist zu beachten, dass zwischen den Realsteuern Substitutionsmöglichkeiten bestehen. Angesichts von Mehrbedarfen können Kommunen gezielt einzelne Realsteuern erhöhen, um eine Anhebung anderer Steuern zu vermeiden. Ebenfalls denkbar ist die Erhöhung einer Realsteuer zur Kompensation von Aufkommensverlusten infolge von Steuersenkungen bei anderen Realsteuern (Postlep 1985, S. 181). Diese Interdependenz dürfte v. a. für die Gewerbesteuer und die Grundsteuer B relevant sein. Bei Vorliegen von 
Mehrbedarfen könnten Kommunen Anreize besitzen, die Grundsteuer B anstelle der Gewerbesteuer zu erhöhen, um ihre Wettbewerbsposition nicht zu verschlechtern (Schwarting 2011, S. 131). Dem ist entgegenzuhalten, dass wiederwahlorientierte Kommunalpolitiker kein Interesse an einer stärkeren Belastung der Bürger haben sollten, um höhere Steuerlasten für Unternehmen zu vermeiden. Schließlich besteht die Gefahr, dass die Bürger ihren Unmut bei den Kommunalwahlen artikulieren und verantwortliche Politiker abstrafen. Grundsätzlich verdeutlichen die Überlegungen jedoch, dass Realsteuern als strategische Substitute fungieren können. Dies legt folgende Hypothese nahe:

$\mathbf{H}_{8}$ Je höher der Hebesatz einer beliebigen Realsteuer, desto niedriger die Hebesätze der anderen Realsteuern.

Überdies bestehen auch ausgabeseitige Wirkmechanismen, die den Hebesatzanspannungsbedarf beeinflussen. Die Generierung von Einnahmen stellt keinen Selbstzweck dar, sondern dient der Refinanzierung notwendiger Ausgaben. Daher ist ein positiver Effekt der Ausgabenseite auf die Hebesätze zu erwarten, da bei gleicher Einnahmensituation und Fehlen alternativer Finanzierungswege höhere Ausgabenbedarfe einen stärkeren Hebesatzanspannungsbedarf bedingen. Dies gilt besonders für Ausgabenkategorien mit Fixkostencharakter, deren Höhe die Kommune kurzfristig nicht autonom beeinflussen kann (Boettcher 2013a; Büttner und Franz 2003). Daraus sind folgende Hypothesen abzuleiten:

H9 Je höher die Gesamtausgaben einer Kommune, desto höher sind ihre Realsteuerhebesätze.

$\mathbf{H}_{10}$ Je höher die Fixkosten einer Kommune, desto höher sind ihre Realsteuerhebesätze.

Eine genauere Analyse der Struktur der Kommunalfinanzen ermöglicht die Identifikation spezifischer Bedarfsträger, die höhere Ausgaben oder sinkende Einnahmen verursachen und daher separat berücksichtigt werden sollen. Beispielsweise impliziert eine hohe, persistente Arbeitslosigkeit einen größeren Hebesatzanspannungsbedarf. Einerseits steigt die Nachfrage nach sozialen Leistungen. Andererseits bedingt hohe Arbeitslosigkeit eine Erosion der Einnahmenseite, da Kommunen mit vielen Arbeitslosen weniger Einnahmen aus den kommunalen Gemeinschaftssteueranteilen erhalten (Kunz 2000, S. 240). Dies führt zu folgender Hypothese:

$\mathbf{H}_{11}$ Je höher die Arbeitslosigkeit, desto höher sind die Realsteuerhebesätze.

Auch die Bevölkerungsstruktur beeinflusst den Hebesatzanspannungsbedarf, da die Nachfrage nach kommunalen Leistungen entlang des Lebenszyklus eines Menschen variiert (Beck 2019, S. 51). So sollte ein hoher Seniorenanteil den Bedarf nach verschiedenen, von Senioren überdurchschnittlich konsumierten Leistungen erhöhen, wodurch die Ausgabenbelastung zunimmt (Bedarfshypothese). Zudem sind Senioren nicht mehr erwerbstätig und erbringen niedrigere Einkommensteuerleistungen. 
Dies schwächt die Einnahmeseite und ist durch die Anpassung anderer Einnahmen zu kompensieren (Ressourcenhypothese). Höhere Ausgaben treffen auf geringere Einnahmen, der Hebesatzanspannungsbedarf steigt (Kunz 2000, S. 233). Ein hoher Anteil von Kindern/Jugendlichen steigert ebenfalls den Hebesatzanspannungsbedarf. Einerseits geht eine junge Bevölkerung mit einer hohen Nachfrage nach Leistungen im Bereich Kinder- und Jugendpolitik einher. Andererseits bedingt sie eine reduzierte Finanzausstattung aufgrund niedrigerer Steuereinnahmen (Kunz 2000, S. 237). Daraus ergeben sich folgende Hypothesen:

$\mathbf{H}_{12}$ Je höher der Anteil von Senioren (Einwohner über 65 Jahre) an der Gesamtbevölkerung, desto höher sind die Realsteuerhebesätze.

$\mathbf{H}_{13}$ Je höher der Anteil von Kindern (Einwohner unter 15 Jahren) an der Gesamtbevölkerung, desto höher sind die Realsteuerhebesätze.

$\mathbf{H}_{14}$ Je höher der Anteil von Jugendlichen und jungen Erwachsenen (Einwohner zwischen 15 und 25 Jahren) an der Gesamtbevölkerung, desto höher sind die Realsteuerhebesätze.

Ein weiterer Parameter der Hebesatzpolitik ist der fiskalische Problemdruck, mit dem Kommunen konfrontiert sind. Dieser äußert sich in Form persistenter Budgetdefizite oder hoher Kreditbestände und beeinflusst die Hebesatzpolitik auf zweierlei Weise. Erstens kann fiskalischer Problemdruck durch gemeindeinterne Entscheidungen Hebesatzerhöhungen induzieren, wenn lokale Entscheider versuchen, die Haushaltskrise mittels Steuererhöhungen zu überwinden (Boettcher 2013a). Zweitens üben staatliche Aufsichtsbehörden massiven Druck aus, die Realsteuerhebesätze anzuspannen, um lokale Haushaltsprobleme zu adressieren (Holtkamp 2000). Dies führt zu folgenden Hypothesen:

$\mathbf{H}_{15}$ Je höher der Finanzierungssaldo (Einnahmen abzüglich Ausgaben), desto geringer sind die Realsteuerhebesätze.

H16 Je höher die Gesamtverschuldung, desto höher sind die Realsteuerhebesätze.

$\mathbf{H}_{17}$ Je höher die Kassenkreditverschuldung, desto höher sind die Realsteuerhebesätze.

\subsection{Der Hebesatzanspannungswille}

Neben dem Hebesatzanspannungspotential und -bedarf dürften auch politische Präferenzen lokaler Entscheidungsträger die Hebesatzpolitik beeinflussen. Die Festlegung der Hebesätze ist letztlich eine politische Entscheidung des Stadtrats, der sich an den Haushaltsplanungen der Verwaltung orientiert. Dabei dürfte der Hebesatzanspannungswille v.a. von der parteipolitischen Affiliation der Ratsmitglieder und des Bürgermeisters abhängen. 
Allgemein nimmt die Parteiendifferenztheorie an, dass die parteipolitische Ausrichtung der Regierung Höhe und Struktur der Besteuerung beeinflusst. Linksparteien als Interessenvertreter von Arbeitnehmern und Personen mit geringem Einkommen präferieren eine starke, progressive Besteuerung, um umfangreiche staatliche Leistungen zu finanzieren, von denen ihre Wählerklientel überproportional profitiert (Garrett 1998, S. 85 ff.; Boix 1998, S. 94-95). Demgegenüber bevorzugen bürgerlich-konservative Parteien als Interessenvertreter der Wirtschaft und von Besserverdienern niedrigere Steuersätze, da sich eine hohe Besteuerung negativ auf ihre Wählerschaft auswirkt (Hallerberg und Basinger 1998, S. 337; Wagschal 2006, S. 271).

Überträgt man die Überlegungen auf die Kommunalpolitik, sollten die Mehrheitsverhältnisse im Stadtrat und die Parteimitgliedschaft des Bürgermeisters den Hebesatzanspannungswillen prägen (Boettcher 2013a; Kunz 2000). Unter der Annahme, dass Linksparteien auch lokal primär die Interessen von Arbeitnehmern und Geringverdienern repräsentieren und umfassende staatliche Leistungen präferieren, sollten sie zu deren Finanzierung eine höhere Besteuerung durchsetzen, Während ihre Wählerschaft von staatlichen Leistungen profitiert, trifft sie die Besteuerung üblicherweise nur marginal. Bürgerliche Parteien sollten hingegen niedrigere Hebesätze bevorzugen, da sich höhere Steuern negativ auf ihr Elektorat auswirken (Person 2020). Diese Zusammenhänge dürften v. a. auf die Gewerbesteuer als wirtschaftsbezogene Kommunalsteuer zutreffen. Im Gegensatz dazu könnte der Wirkmechanismus bei der Grundsteuer B möglicherweise nur eingeschränkt gelten, da in Deutschland eine Umlage der Grundsteuer auf die Mietkosten zulässig ist. Findet eine solche Überwälzung auf die Mieter statt, wirken sich Erhöhungen der Grundsteuer negativ auf eine wichtige Zielgruppe linker Parteien (Geringverdiener) aus. Dies schränkt gegebenenfalls ihre Bereitschaft zu Steuererhöhungen bei der Grundsteuer B ein. Aus den vorgenannten Überlegungen ergeben sich somit folgende Hypothesen:

$\mathbf{H}_{18 a}$ Verfügen bürgerlich-konservative Parteien (CDU, FDP) über eine Mehrheit der Sitze im Stadtrat, wirkt sich diese Mehrheit negativ auf die Höhe der Realsteuerhebesätze aus.

$\mathbf{H}_{\mathbf{1 8 b}}$ Verfügen linke Parteien (SPD, Grüne, Linkspartei) über eine Mehrheit im Stadtrat, wirkt sich diese Mehrheit positiv auf die Höhe der Realsteuerhebesätze aus.

$\mathbf{H}_{19 a}$ Gehört der Bürgermeister einer bürgerlich-konservativen Partei an, fallen die Realsteuerhebesätze niedriger aus.

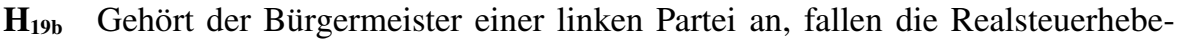
sätze höher aus.

Überdies weisen politökonomische Ansätze auf die Zersplitterung politischer Entscheidungsgremien als Einflussfaktor für die haushaltspolitische Handlungsfähigkeit einer Gebietskörperschaft hin. Eine hohe Fragmentierung bedingt einen intensiveren 
Tab. 1 Übersicht über potenzielle Determinanten der Realsteuerhebesätze

\begin{tabular}{|c|c|c|c|c|c|}
\hline \multirow{2}{*}{$\begin{array}{l}\text { Hypo- } \\
\text { these }\end{array}$} & \multirow[t]{2}{*}{ Variable } & \multirow[t]{2}{*}{ Indikator/Operationalisierung } & \multicolumn{3}{|c|}{ Wirkrichtung } \\
\hline & & & $\begin{array}{l}\text { Gewerbe- } \\
\text { steuer }\end{array}$ & $\begin{array}{l}\text { Grund- } \\
\text { steuer A }\end{array}$ & $\begin{array}{l}\text { Grund- } \\
\text { steuer B }\end{array}$ \\
\hline \multirow[t]{3}{*}{$\mathrm{H}_{1}$} & Grundbetrag Gewerbesteuer & $€ /$ Einwohner & + & 0 & 0 \\
\hline & Grundbetrag Grundsteuer A & $€ /$ Einwohner & 0 & + & 0 \\
\hline & Grundbetrag Grundsteuer B & $€ /$ Einwohner & 0 & 0 & + \\
\hline $\mathrm{H}_{2}$ & Arbeitsplatzzentralität & $\begin{array}{l}\text { Pendlersaldo/Einwohner im } \\
\text { erwerbsfähigen Alter (in \%) }\end{array}$ & + & + & + \\
\hline $\mathrm{H}_{3}$ & Bevölkerungsdichte & $\begin{array}{l}\text { Einwohnerzahl/Bodenfläche in } \\
\text { Hektar (in \%) }\end{array}$ & + & + & + \\
\hline $\mathrm{H}_{4}$ & Arbeitslosenquote & $\begin{array}{l}\text { Registrierte Arbeitslose/ } \\
\text { Einwohner im erwerbsfähigen } \\
\text { Alter (in \%) }\end{array}$ & - & - & - \\
\hline $\mathrm{H}_{5}$ & Gemeindegröße & $\begin{array}{l}\text { Natürlicher Logarithmus der } \\
\text { Einwohnerzahl }\end{array}$ & + & + & + \\
\hline $\mathrm{H}_{6}$ & Finanzkraft & $\begin{array}{l}\text { Summe aus Grundbetrag der Real- } \\
\text { steuern, Einkommens-/Umsatz- } \\
\text { steueranteil und allgemeine Zu- } \\
\text { weisungen (in } € / \text { Einwohner) }\end{array}$ & - & - & - \\
\hline \multirow[t]{3}{*}{$\mathrm{H}_{7}$} & Grundbetrag Gewerbesteuer & $€ /$ Einwohner & - & 0 & 0 \\
\hline & Grundbetrag Grundsteuer A & $€ /$ Einwohner & 0 & - & 0 \\
\hline & Grundbetrag Grundsteuer B & $€ /$ Einwohner & 0 & 0 & - \\
\hline \multirow[t]{3}{*}{$\mathrm{H}_{8}$} & Hebesatz Gewerbesteuer & In Prozent & 0 & - & - \\
\hline & Hebesatz Grundsteuer A & In Prozent & - & 0 & - \\
\hline & Hebesatz Grundsteuer B & In Prozent & - & - & 0 \\
\hline $\mathrm{H}_{9}$ & Gesamtausgaben & $€ /$ Einwohner & + & + & + \\
\hline $\mathrm{H}_{10}$ & Fixkosten & $\begin{array}{l}\text { Summe aus Ausgaben für } \\
\text { Soziales, Zinsen, Umlagen (in } \\
€ / \text { Einwohner) }\end{array}$ & + & + & + \\
\hline $\mathrm{H}_{11}$ & Arbeitslosenquote & $\begin{array}{l}\text { Registrierte Arbeitslose/Einwoh- } \\
\text { ner im erwerbsfähigen Alter (in \%) }\end{array}$ & + & + & + \\
\hline $\mathrm{H}_{12}$ & Seniorenquote & $\begin{array}{l}\text { Anzahl der Einwohner über } \\
65 \text { Jahre/Einwohnerzahl (in \%) }\end{array}$ & + & + & + \\
\hline $\mathrm{H}_{13}$ & $\begin{array}{l}\text { Anteil Kinder an der Ge- } \\
\text { samtbevölkerung }\end{array}$ & $\begin{array}{l}\text { Anzahl der Einwohner unter } \\
15 \text { Jahren/Einwohnerzahl (in \%) }\end{array}$ & + & + & + \\
\hline $\mathrm{H}_{14}$ & $\begin{array}{l}\text { Anteil Jugendliche/junge } \\
\text { Erwachsene an der Gesamt- } \\
\text { bevölkerung }\end{array}$ & $\begin{array}{l}\text { Anzahl der Einwohner zwi- } \\
\text { schen } 15 \text { und } 25 \text { Jahren/ } \\
\text { Einwohnerzahl (in \%) }\end{array}$ & + & + & + \\
\hline $\mathrm{H}_{15}$ & Finanzierungssaldo & $€ /$ Einwohner & - & - & - \\
\hline $\mathrm{H}_{16}$ & Gesamtverschuldung & $€ /$ Einwohner & + & + & + \\
\hline $\mathrm{H}_{17}$ & Kassenkredite & $€ /$ Einwohner & + & + & + \\
\hline $\mathrm{H}_{18 \mathrm{a}}$ & $\begin{array}{l}\text { Mehrheit bürgerlich-konser- } \\
\text { vativer Parteien }\end{array}$ & Dummy-Variable $(1=$ ja $)$ & - & - & - \\
\hline $\mathrm{H}_{18 \mathrm{~b}}$ & Mehrheit linker Parteien & Dummy-Variable $(1=$ ja $)$ & + & + & + \\
\hline $\mathrm{H}_{19 \mathrm{a}}$ & $\begin{array}{l}\text { Bürgerlich-konservativer } \\
\text { Bürgermeister }\end{array}$ & Dummy-Variable $(1=$ ja $)$ & - & - & - \\
\hline $\mathrm{H}_{19 b}$ & Linker Bürgermeister & Dummy-Variable $(1=$ ja $)$ & + & + & + \\
\hline $\mathrm{H}_{20}$ & $\begin{array}{l}\text { Fragmentierung des Ge- } \\
\text { meinderates }\end{array}$ & Rae-Index & + & + & + \\
\hline
\end{tabular}


Wettbewerb zwischen politischen Gruppen, der sich in einer stärkeren Konkurrenz um öffentliche Güter und höheren Ausgabelasten niederschlägt, da zahlreiche Partikularinteressen bedient werden müssen. Gleichzeitig wird die Kompromissfindung erschwert, da mehr Akteure zum Erreichen politischer Mehrheiten benötigt werden. Dadurch werden umfassende Verhandlungspakete notwendig. Dies führt zu einem wachsenden Finanzbedarf, der über höhere Abgaben oder Kredite gedeckt werden muss (Alesina und Perotti 1995; Wagschal 2005, S. 401ff.). Allerdings begrenzt auf der lokalen Ebene das Gemeindehaushaltsrecht und die staatliche Haushaltsaufsicht die Möglichkeit einer Kreditfinanzierung höherer Ausgabenbedarfe. Folglich bleibt nur der Weg einer Refinanzierung über höhere Abgaben. Dies führt zu folgender Hypothese:

$\mathbf{H}_{20}$ Je stärker die Fragmentierung des Stadtrates, desto höher sind die Realsteuerhebesätze.

Eine zusammenfassende Übersicht über die potentiellen Determinanten der Realsteuerhebesätze, ihrer Operationalisierung im Rahmen der empirischen Analyse sowie ihrer erwarteten Wirkrichtung bietet Tabelle 1.

\section{Daten und Forschungsdesign}

Um die Hypothesen zu prüfen, wird die Hebesatzpolitik in sechs Bundesländern zwischen 2004 und 2013 analysiert. ${ }^{2}$ Die betrachteten Länder sind: Baden-Württemberg, Bayern, Hessen, Nordrhein-Westfalen, Rheinland-Pfalz und Thüringen. Für die übrigen Länder standen die erforderlichen Daten nicht in ausreichender Form zur Verfügung. Trotz der Beschränkung des Samples sollte eine angemessene Repräsentativität gegeben sein. Erstens erfasst das Sample eine Mehrheit der Kommunen Deutschlands (je nach Jahr 59-64\%) und deckt 65\% der Gesamtbevölkerung ab. Zweitens werden regionale Unterschiede bezüglich der sozioökonomischen Situation einzelner Landesteile berücksichtigt, da wirtschaftsstarke und strukturschwächere Länder betrachtet werden. Auch Ost-West-Unterschiede finden partiell Berücksichtigung. Drittens trägt das Sample unterschiedlichen Gemeindestrukturen Rechnung. Es enthält sowohl Länder mit einer kleinteiligen Gemeindestruktur (Baden-Württemberg, Bayern, Rheinland-Pfalz) als auch Länder mit eher größeren Kommunen (Hessen, Nordrhein-Westfalen). Viertens weisen die Länder unterschiedliche kommunalpolitische Rahmenbedingungen auf, die den Grad der Parteipolitisierung der Lokalpolitik beeinflussen (Holtkamp 2008). Dadurch werden konkurrenz- und konkordanzdemokratische Systeme erfasst.

\footnotetext{
2 Die zeitliche Einschränkung des Untersuchungszeitraums ist primär der Datenverfügbarkeit geschuldet. Einerseits lagen zum Zeitpunkt der Datenerhebung keine aktuelleren Daten in gemeindescharfer Abgrenzung vor. Andererseits konnten für einige Länder ältere Daten nicht oder nur eingeschränkt bereitgestellt werden. Eine Besonderheit ist zudem bei der Gewerbesteuer zu beachten: Seit 2004 müssen Kommunen einen Mindesthebesatz erheben. Dieser Strukturbruch schränkt die Vergleichbarkeit der Daten vor und nach 2004 ein.
} 
Um die Effekte einzelner Erklärungsfaktoren auf die Hebesatzpolitik zu erfassen, werden lineare OLS-Regressionsverfahren für Panel-Daten verwendet. Der datengenerierende Prozess wird durch folgendes Modell beschrieben:

$$
y_{i t}=\alpha_{i}+\gamma_{t}+\delta_{1} d_{\mathrm{LRit}-1}+\delta_{2} d_{\mathrm{RRit}-1}+\delta_{3} d_{\mathrm{LBit}-1}+\delta_{4} d_{\mathrm{RBit}-1}+\boldsymbol{x}_{i t-1}^{\prime} \boldsymbol{\beta}+\epsilon_{i t}
$$

Die abhängige Variable $y_{i t}$ erfasst den Realsteuerhebesatz in Kommune i zum Zeitpunkt t. Die Dummy-Variablen $d_{\text {LRit-1 }}$ und $d_{\text {RRit-1 }}$ zeigen an, ob linke bzw. bürgerliche Parteien die Sitzmehrheit im Stadtrat innehaben. Die Basiskategorie sind Situationen, in denen kein politisches Lager die Mehrheit besitzt. Die Dummy-Variablen $d_{\mathrm{LBit}-1}$ und $d_{\mathrm{RBit}-1}$ geben an, ob der Bürgermeister einer linken oder bürgerlich-konservativen Partei angehört. Referenzkategorie sind parteiungebundene

Tab. 2 Deskriptive Statistik der abhängigen und unabhängigen Variablen

\begin{tabular}{|c|c|c|c|c|}
\hline Variable & Mittelwert & Std. Abw & Minimum & Maximum \\
\hline Hebesatz Gewerbesteuer (in \%) & 342,79 & 33,03 & 200,00 & 600,00 \\
\hline Hebesatz Grundsteuer A (in \%) & 304,05 & 72,23 & 0,00 & 1800,00 \\
\hline Hebesatz Grundsteuer B (in \%) & 332,25 & 49,07 & 0,00 & 825,00 \\
\hline Grundbetrag Gewerbesteuer ( $€ /$ Einwohner) & 72,67 & 108,40 & $-1055,56$ & 2853,70 \\
\hline Grundbetrag Grundsteuer A (€/Einwohner) & 3,22 & 3,07 & $-29,52$ & 62,50 \\
\hline Grundbetrag Grundsteuer B ( $€ /$ Einwohner) & 25,90 & 9,93 & $-86,91$ & 639,05 \\
\hline Arbeitsplatzzentralität (in \%) & $-22,71$ & 26,23 & $-205,77$ & 450,38 \\
\hline Bevölkerungsdichte (in \%) & 2,28 & 3,04 & 0,04 & 44,68 \\
\hline Arbeitslosenquote (in \%) & 4,36 & 2,65 & 0,00 & 27,08 \\
\hline $\begin{array}{l}\text { Gemeindegröße (Einwohnerzahl, logarith- } \\
\text { miert) }\end{array}$ & 7,87 & 1,38 & 2,30 & 14,14 \\
\hline Finanzkraft (€/Einwohner) & 638,86 & 188,64 & $-282,29$ & 3814,93 \\
\hline Gesamtausgaben ( $€$ /Einwohner) & 1653,43 & 757,58 & 0,00 & 9744,65 \\
\hline Kommunale Fixkosten ( $€ /$ Einwohner) & 554,45 & 224,26 & 0,00 & 4720,47 \\
\hline Anteil Senioren (in \%) & 19,28 & 3,40 & 3,97 & 47,06 \\
\hline Anteil Kinder (in \%) & 14,74 & 2,57 & 0,00 & 33,53 \\
\hline Anteil Jugendliche/junge Erwachsene (in \%) & 11,60 & 1,71 & 0,00 & 23,55 \\
\hline Finanzierungssaldo ( $€$ /Einwohner) & 35,56 & 295,69 & $-4391,63$ & 4866,40 \\
\hline $\begin{array}{l}\text { Höhe der Gesamtverschuldung ( } € / \\
\text { Einwohner) }\end{array}$ & 591,94 & 629,03 & 0,00 & $11.899,61$ \\
\hline Höhe der Kassenkredite ( $€ /$ Einwohner) & 57,76 & 276,19 & 0,00 & 6983,17 \\
\hline $\begin{array}{l}\text { Bürgerlich-konservative Mehrheit im Rat } \\
\text { (Dummy: } 1=\mathrm{ja} \text { ) }\end{array}$ & 0,15 & 0,36 & 0,00 & 1,00 \\
\hline Linke Mehrheit im Rat (Dummy: 1= ja) & 0,05 & 0,22 & 0,00 & 1,00 \\
\hline $\begin{array}{l}\text { Bürgerlich-konservativer Bürgermeister } \\
\text { (Dummy: } 1=\mathrm{ja} \text { ) }\end{array}$ & 0,36 & 0,48 & 0,00 & 1,00 \\
\hline Linker Bürgermeister (Dummy: $1=\mathrm{ja}$ ) & 0,15 & 0,36 & 0,00 & 1,00 \\
\hline Fragmentierung des Gemeinderates (Rae-Index) & 0,42 & 0,28 & 0,00 & 0,82 \\
\hline
\end{tabular}

Alle Daten stammen, soweit nicht anders angeben, von den Statistischen Ämtern des Bundes und der Länder

Pendler- und Arbeitslosendaten wurden von der Bundesagentur für Arbeit bezogen

Bürgermeisterdaten stammen von den Landeswahlleitern oder wurden eigenständig erhoben 
Amtsinhaber. Der Spaltenvektor $\boldsymbol{x}_{i t-1}$ enthält die übrigen zeitveränderlichen Prädiktoren. Die unabhängigen Variablen weisen einen time-lag von einem Jahr auf, da die Festlegung des Hebesatzes für ein Jahr t üblicherweise im Rahmen der Haushaltsberatungen gegen Ende des Vorjahres t-1 erfolgt. Zudem enthält das Modell eine zeitinvariante einheitsspezifische Konstante $\alpha_{i}$ (unit-specific fixed effects), die für unbeobachtete Heterogenität kontrolliert, sowie fixe Zeiteffekte $\gamma_{t}$ (time-specific fixed effects), die für kommuneneinheitliche Unterschiede über die Zeit kontrollieren (z. B. Veränderungen, die simultan alle Kommunen betreffen). Letztere erfassen beispielsweise die Auswirkungen makroökonomischer Schocks, die mit einer gewissen zeitlichen Verzögerung auf die Kommunalfinanzen durchschlagen, wie den Konjunktureinbruch zu Beginn des Jahrtausends oder die globale Finanz- und Wirtschaftskrise 2008/2009. $\epsilon_{i t}$ ist ein unabhängig und identisch verteilter Fehlerterm. Zur Schätzung des Modells werden Fixed-Effects-Schätzer verwendet, da HausmanTests eine Verletzung der Random-Effects-Annahme anzeigen. Folglich liefert ein Random-Effects-Modell verzerrte Parameterschätzungen. Um für unterschiedliche Fehlervarianzen zwischen Untersuchungseinheiten (Heteroskedastizität) und seriell korrelierte Fehlerterme in Untersuchungseinheiten (Autokorrelation) zu korrigieren, werden panel-robuste Standardfehler verwendet.

Einen Überblick über die Daten bietet Tab. 2. Insgesamt werden 57.475 Beobachtungen (Kommunen-Jahre) verwendet, die in 7056 Untersuchungseinheiten (Kommunen) geclustert sind. Zwar enthielt das Sample ursprünglich mehr Beobachtungen (72.320). Allerdings wiesen manche Beobachtungen bei einzelnen Variablen erhebliche Ausreißer oder unplausible Werte auf, die bereinigt wurden. Dies reduzierte das Sample um 116 Beobachtungen. Nach Ausschluss fehlender Werte verringerte sich die Zahl der Beobachtungen um 14.729 auf 57.475. Dabei ist zu beachten, dass durch die Verwendung des einjährigen time-lags das erste Untersuchungsjahr entfällt, wodurch alleine 7361 Beobachtungen ausgeschlossen werden. Die übrigen Ausschlüsse entstehen primär durch die Variable Arbeitsplatzzentralität, da in der Pendlerstatistik Werte kleiner Drei anonymisiert werden, wodurch diese Beobachtungen als missings codiert werden mussten.

\section{Empirische Analyse}

Die Ergebnisse der Regressionsanalyse finden sich in Tab. 3. Für jene Variablen, die das Hebesatzanspannungspotential erfassen, zeigen sich gemischte Befunde. Im Einklang mit Hypothese $\mathrm{H}_{4}$ lässt sich ein negativer Effekt der Arbeitslosenquote feststellen: Steigt diese um einen Prozentpunkt, sinken die Hebesätze um ein bis zwei Punkte. Am stärksten ist der Effekt bei der Gewerbesteuer. Angesichts wachsender Arbeitslosigkeit scheinen die Hebesätze somit tatsächlich als Instrument zur Steigerung der Wettbewerbsfähigkeit zu fungieren (Büttner und Franz 2003). Anders als erwartet zeigt sich auch für die Gemeindegröße ein negativer Effekt ${ }^{3}$ : Ein Anstieg der Einwohnerzahl geht mit einer Absenkung der Hebesätze einher. Dies könnte

\footnotetext{
3 Verwendet man die tatsächliche Einwohnerzahl, bleibt der negative Koeffizient erhalten, wird jedoch insignifikant (ausgenommen Grundsteuer A).
} 
Tab. 3 Determinanten der Realsteuerhebesatzpolitik (2004-2013)

\begin{tabular}{|c|c|c|c|}
\hline & $\begin{array}{l}\text { Modell 1a } \\
\text { Gewerbesteuer }\end{array}$ & $\begin{array}{l}\text { Modell } 1 \mathrm{~b} \\
\text { Grundsteuer A }\end{array}$ & $\begin{array}{l}\text { Modell 1c } \\
\text { Grundsteuer B }\end{array}$ \\
\hline $\begin{array}{l}\text { Grundbetrag Gewerbesteuer } \\
\text { (€/Einwohner) }\end{array}$ & $\begin{array}{c}-0,008 * \\
(0,002)\end{array}$ & & \\
\hline $\begin{array}{l}\text { Grundbetrag Grundsteuer A } \\
(€ / \text { Einwohner) }\end{array}$ & & $\begin{array}{c}0,065 \\
(0,101)\end{array}$ & \\
\hline $\begin{array}{l}\text { Grundbetrag Grundsteuer B } \\
(€ / \text { Einwohner) }\end{array}$ & & & $\begin{array}{l}-0,030 \\
(0,024)\end{array}$ \\
\hline Arbeitsplatzzentralität (in \%) & $\begin{array}{l}-0,040 \\
(0,023)\end{array}$ & $\begin{array}{l}-0,016 \\
(0,022)\end{array}$ & $\begin{array}{c}0,002 \\
(0,025)\end{array}$ \\
\hline Bevölkerungsdichte (in \%) & $\begin{array}{c}2,521 \\
(1,689)\end{array}$ & $\begin{array}{c}2,322 \\
(1,667)\end{array}$ & $\begin{array}{l}-1,272 \\
(1,803)\end{array}$ \\
\hline Arbeitslosenquote (in \%) & $\begin{array}{c}-1,818 * \\
(0,102)\end{array}$ & $\begin{array}{r}-0,844 * \\
(0,115)\end{array}$ & $\begin{array}{c}-0,791 * \\
(0,120)\end{array}$ \\
\hline $\begin{array}{l}\text { Gemeindegröße (Einwohnerzahl, } \\
\text { logarithmiert) }\end{array}$ & $\begin{array}{r}-24,832 * \\
(4,271)\end{array}$ & $\begin{array}{r}-20,808 * \\
(5,054)\end{array}$ & $\begin{array}{r}-23,394 * \\
(5,523)\end{array}$ \\
\hline Finanzkraft ( $€ /$ Einwohner) & $\begin{array}{c}-0,002 * \\
(0,001)\end{array}$ & $\begin{array}{c}-0,002 * \\
(0,001)\end{array}$ & $\begin{array}{c}-0,005^{*} \\
(0,001)\end{array}$ \\
\hline Hebesatz Gewerbesteuer (in \%) & & $\begin{array}{c}0,107^{*} \\
(0,019)\end{array}$ & $\begin{array}{c}0,195^{*} \\
(0,024)\end{array}$ \\
\hline Hebesatz Grundsteuer A (in \%) & $\begin{array}{c}0,093^{*} \\
(0,015)\end{array}$ & & $\begin{array}{r}0,460 * \\
(0,034)\end{array}$ \\
\hline Hebesatz Grundsteuer B (in \%) & $\begin{array}{c}0,141 * \\
(0,014)\end{array}$ & $\begin{array}{c}0,428 * \\
(0,021)\end{array}$ & \\
\hline Gesamtausgaben ( $€ /$ Einwohner) & $\begin{array}{r}-0,000 \\
(0,000)\end{array}$ & $\begin{array}{r}-0,001 * \\
(0,000)\end{array}$ & $\begin{array}{c}-0,003 * \\
(0,000)\end{array}$ \\
\hline Fixkosten ( $€ /$ Einwohner) & $\begin{array}{c}0,002 * \\
(0,001)\end{array}$ & $\begin{array}{c}-0,001 \\
(0,001)\end{array}$ & $\begin{array}{c}-0,003 * \\
(0,001)\end{array}$ \\
\hline Seniorenquote (in \%) & $\begin{array}{r}0,288 * \\
(0,120)\end{array}$ & $\begin{array}{c}-0,014 \\
(0,137)\end{array}$ & $\begin{array}{c}0,046 \\
(0,157)\end{array}$ \\
\hline Anteil Kinder (in \%) & $\begin{array}{c}0,879 * \\
(0,123)\end{array}$ & $\begin{array}{c}0,585^{*} \\
(0,159)\end{array}$ & $\begin{array}{l}1,297 * \\
(0,175)\end{array}$ \\
\hline $\begin{array}{l}\text { Anteil Jugendliche/junge Erwachse- } \\
\text { ne (in \%) }\end{array}$ & $\begin{array}{r}-1,192 * \\
(0,113)\end{array}$ & $\begin{array}{c}-0,813^{*} \\
(0,136)\end{array}$ & $\begin{array}{c}-1,109 * \\
(0,154)\end{array}$ \\
\hline Finanzierungssaldo ( $€$ /Einwohner) & $\begin{array}{c}0,001^{*} \\
(0,000)\end{array}$ & $\begin{array}{c}-0,000 \\
(0,000)\end{array}$ & $\begin{array}{l}-0,000 \\
(0,000)\end{array}$ \\
\hline Gesamtverschuldung ( $€ /$ Einwohner) & $\begin{array}{c}0,002 * \\
(0,000)\end{array}$ & $\begin{array}{r}0,002^{*} \\
(0,001)\end{array}$ & $\begin{array}{l}0,004^{*} \\
(0,001)\end{array}$ \\
\hline Kassenkredite ( $€ /$ Einwohner) & $\begin{array}{c}0,006^{*} \\
(0,001)\end{array}$ & $\begin{array}{l}0,010^{*} \\
(0,001)\end{array}$ & $\begin{array}{c}0,021 * \\
(0,002)\end{array}$ \\
\hline Bürgerliche Mehrheit im Rat & $\begin{array}{r}-0,100 \\
(0,560)\end{array}$ & $\begin{array}{c}-1,646^{*} \\
(0,737)\end{array}$ & $\begin{array}{r}-0,647 \\
(0,741)\end{array}$ \\
\hline Linke Mehrheit im Rat & $\begin{array}{r}-0,402 \\
(0,805)\end{array}$ & $\begin{array}{l}-1,603 \\
(1,472)\end{array}$ & $\begin{array}{l}3,911^{*} \\
(1,411)\end{array}$ \\
\hline $\begin{array}{l}\text { Bürgerlich-konservativer Bürger- } \\
\text { meister }\end{array}$ & $\begin{array}{c}0,066 \\
(0,574)\end{array}$ & $\begin{array}{c}0,062 \\
(0,628)\end{array}$ & $\begin{array}{r}-0,252 \\
(0,753)\end{array}$ \\
\hline Linker Bürgermeister & $\begin{array}{r}-1,067 \\
(0,628)\end{array}$ & $\begin{array}{r}-1,022 \\
(0,834)\end{array}$ & $\begin{array}{l}-1,128 \\
(0,882)\end{array}$ \\
\hline
\end{tabular}


Tab. 3 (Fortsetzung)

\begin{tabular}{llcc}
\hline & $\begin{array}{l}\text { Modell 1a } \\
\text { Gewerbesteuer }\end{array}$ & $\begin{array}{c}\text { Modell 1b } \\
\text { Grundsteuer A }\end{array}$ & $\begin{array}{c}\text { Modell 1c } \\
\text { Grundsteuer B }\end{array}$ \\
\hline Fragmentierung des Gemeinderats & $-6,647^{*}$ & 0,689 & $-0,918$ \\
(Rae-Index) & $(1,991)$ & $(2,022)$ & $(2,124)$ \\
\hline $\mathrm{R}^{2}$ (Within) & 0,350 & 0,387 & 0,478 \\
Anzahl Beobachtungen & 57.475 & 57.475 & 57.475 \\
Anzahl Untersuchungseinheiten & 7056 & 7056 & 7056 \\
\hline
\end{tabular}

Dargestellt sind unstandardisierte Regressionskoeffizienten (panel-robuste Standardfehler in Klammern) Alle Modelle enthalten time-Dummies für einzelne Untersuchungsjahre (Basisjahr: 2005; nicht dargestellt) $* p<0,05$ (zweiseitiger Test)

daran liegen, dass wachsende Kommunen häufig ökonomisch prosperieren. Verbessert sich ihre Finanzsituation, lassen sie möglicherweise Bürger und Unternehmen durch Steuersenkungen daran teilhaben. ${ }^{4}$ Demgegenüber sind die Arbeitsplatzzentralität und die Bevölkerungsdichte nicht erklärungskräftig. Beide Variablen weisen unterschiedliche Vorzeichen auf, die auch zwischen den Realsteuern divergieren. Außerdem sind die Effekte insignifikant. Die Hypothesen $\mathrm{H}_{2}$ und $\mathrm{H}_{3}$ sind somit zurückzuweisen. Auch die Realsteuergrundbeträge sind nur bedingt erklärungskräftig. Lediglich der Gewerbesteuergrundbetrag weist einen statistisch signifikanten, negativen Effekt auf: Ein Anstieg des Grundbetrages geht mit einer leichten Reduktion des Gewerbesteuerhebesatzes einher. Kommunen scheinen die ökonomische Leistungsfähigkeit örtlicher Unternehmen nicht auszunutzen, um höhere Steuersätze festzulegen, sondern nutzen die breitere Steuerbasis, um Unternehmen zu schonen und Hebesätze abzusenken. Somit ist Hypothese $\mathrm{H}_{1}$ generell, Hypothese $\mathrm{H}_{8}$ bezüglich der Grundsteuern, zu verwerfen.

Mit Blick auf den Hebesatzanspannungsbedarf zeigen sich ebenfalls gemischte Befunde. Im Einklang mit Hypothese $\mathrm{H}_{6}$ lässt sich ein signifikanter, negativer Zusammenhang zwischen Finanzkraft und Hebesatzniveau beobachten: Erhöhen sich die Einnahmekapazitäten einer Kommune, sinken die Hebesätze leicht. Demgegenüber keine Bestätigung findet die Annahme, dass Realsteuern als strategische Substitute fungieren. Wäre dies der Fall, müsste zwischen den Hebesätzen ein negativer Zusammenhang bestehen. Im Widerspruch zu Hypothese $\mathrm{H}_{8}$ zeigen sich jedoch stets positive Zusammenhänge: Eine Hebesatzerhöhung bei einer beliebigen Realsteuer geht mit Anstiegen bei den anderen einher. Besonders ausgeprägt ist dies bei den Grundsteuern. Dieser Befund deckt sich mit den Ergebnissen von Beck (2019, S. 48-49) und lässt sich dadurch erklären, dass Steuersatzänderungen die allgemeinen steuerpolitischen Präferenzen lokaler Entscheider reflektieren. Neigen diese zu einer expansiven/restriktiven Steuerpolitik oder sehen sich aufgrund äußerer Umstände zu Steuersatzanpassungen gezwungen, sollte sich dies bei allen Realsteuern manifestieren.

\footnotetext{
${ }^{4}$ Da Fixed-Effects-Modelle nur die Längsschnitt-, nicht die Querschnitts-Varianz, in den Daten berücksichtigen, kann der Koeffizient nicht so interpretiert werden, dass größere Städte niedrigere Hebesätze aufweisen.
} 
Ambivalent fallen die Ergebnisse bezüglich der Ausgabenvariablen aus. Während sich im Einklang mit Hypothese $\mathrm{H}_{10}$ bei der Gewerbesteuer ein signifikanter, positiver Effekt der Fixkosten zeigt, entfalten diese bei den Grundsteuern einen negativen Effekt, der jedoch nur bei der Grundsteuer B signifikant ist. Im Widerspruch zu Hypothese $\mathrm{H}_{9}$ lassen sich für die Gesamtausgaben sogar durchgängig negative Effekte beobachten, die nur bei der Gewerbesteuer insignifikant sind. Anders als erwartet führt wachsender Ausgabendruck nicht automatisch zu höheren Hebesätzen, da Kommunen vielleicht versuchen, zunehmende Ausgaben anderweitig zu finanzieren, um eine übermäßige Belastung der Steuerzahler zu vermeiden.

Ebenfalls widersprüchliche Befunde liefern die Bevölkerungsstrukturvariablen. Im Einklang mit Hypothese $\mathrm{H}_{13}$ weist die Kinderquote einen signifikanten, positiven Effekt auf die Hebesätze auf: Steigt die Kinderquote um einen Punkt, erhöhen sich die Hebesätze um einen halben bis einen Punkt. Auch ein Anstieg der Seniorenquote führt zu höheren Hebesätzen. Allerdings ist der Effekt schwächer und nur bei der Gewerbesteuer signifikant. Entgegen den Erwartungen zeigt sich für den Anteil an Personen zwischen 15 und 25 Jahren ein negativer Effekt. Möglicherweise ist bei dieser Gruppe die Nachfrage nach kommunalen Leistungen schwächer ausgeprägt. Außerdem leisten Mitglieder dieser Gruppe, die schon am Berufsleben partizipieren, einen Beitrag zu den kommunalen Einnahmequellen, v. a. den Gemeinschaftssteueranteilen, was sich hebesatzbegrenzend auswirken könnte.

Interessante Ergebnisse bieten auch die Schuldenvariablen. Im Einklang mit Hypothese $\mathrm{H}_{16}$ und $\mathrm{H}_{17}$ geht ein Anstieg der Verschuldung mit höheren Hebesätzen einher. Dies gilt für die kurzfristige Verschuldung (Kassenkredite) wie die Gesamtverschuldung, wobei der Effekt der Kassenkreditverschuldung deutlich stärker ausfällt. Zudem sind die Effekte bei der Grundsteuer B ausgeprägter als bei den übrigen Realsteuern, insbesondere der Gewerbesteuer. Dies verdeutlicht, dass angesichts hohen fiskalischen Problemdrucks häufig eine Anpassung der Steuersätze zur Konsolidierung der Haushalte vorgenommen wird, vorzugsweise bei der Grundsteuer B. Dies dürfte daran liegen, dass man aufgrund der Immobilität der Bemessungsgrundlage weniger schädliche Auswirkungen auf die Wettbewerbsfähigkeit fürchtet als bei der Gewerbesteuer. Ambivalenter sind die Effekte des Finanzierungssaldos. Während diese bei den Grundsteuern insignifikant sind, zeigt sich bei der Gewerbesteuer wider Erwarten ein signifikanter, positiver Effekt. Demnach geht eine Verbesserung des Finanzierungssaldos mit höheren Gewerbesteuerhebesätzen einher. Dies überrascht, da man erwarten würde, dass Kommunen, deren Haushaltslage sich verschlechtert, höhere Hebesätze festsetzen. Möglicherweise spiegeln sich hierin langfristige Konsolidierungseffekte. Waren Kommunen in der Vergangenheit mit Haushaltsproblemen konfrontiert, bekämpften sie diese vermutlich mit Steuererhöhungen, wodurch sich in den Folgejahren Budgetüberschüsse ergaben. Wurden die Hebesätze nach erfolgreicher Haushaltskonsolidierung nicht wieder reduziert, erklärt dies die unerwartete Effektrichtung (Person 2020, S. 77). Um dies zu prüfen, wurde der Finanzierungssaldo mit längeren time-lags in die Modelle integriert. Ab einer Verzögerung von drei und mehr Jahren wird der Effekt negativ.

Auch politische Faktoren sind nur begrenzt erklärungskräftig. Die Parteizugehörigkeit des Bürgermeisters zeigt oft unerwartete Effekte: So geht der Wechsel von parteilosen zu linken Bürgermeistern mit einer Reduktion der Hebesätze einher, 
die Amtsübernahme eines konservativen Verwaltungschefs mit einem Anstieg (Ausnahme: Grundsteuer B). Die Effekte sind jedoch stets insignifikant. Demgegenüber sind für die parteipolitische Ausrichtung des Stadtrats vereinzelt signifikante Effekte beobachtbar. Bei der Grundsteuer A führt der Übergang von unklaren Mehrheitsverhältnissen zu bürgerlichen Ratsmehrheiten zu einer Senkung der Hebesätze um 1,6 Punkte. Überraschend entfalten auch linke Mehrheiten einen negativen Effekt, der jedoch insignifikant ist. Noch mehr im Einklang mit den Erwartungen sind die Befunde für die Grundsteuer B: Während der Wechsel zu bürgerlichen Mehrheiten mit einer Hebesatzsenkung um 0,6 Punkte einhergeht, bewirkt der Übergang zu linken Mehrheiten einen Anstieg um vier Punkte, wobei nur letzterer signifikant ist. Entgegen den Überlegungen im Theorieteil scheint die bloße Möglichkeit, die Kosten der Grundsteuer über die Nebenkostenabrechnung auf die Mieter zu überwälzen, linke Parteien nicht per se von einer Erhöhung der Grundsteuer abzuhalten, selbst wenn sich diese gegebenenfalls negativ auf eine ihrer Wählergruppen (Geringverdiener) auswirkt. Auch die Fragmentierung des Stadtrats ist meist kein relevanter Erklärungsfaktor. Nur bei der Gewerbesteuer ist ein signifikanter Effekt beobachtbar. Dieser fällt wider Erwarten negativ aus: Eine zunehmende Zersplitterung des Rats bewirkt demnach eine Senkung des Hebesatzes.

Allerdings ist zu beachten, dass der Grad der Parteipolitisierung der Lokalpolitik zwischen den Ländern variiert, da eine enge Wechselwirkung zwischen der Ausgestaltung der Kommunalverfassung und lokalen Entscheidungsprozessen besteht, mithin von der Gemeindeordnung entpolitisierende Effekte ausgehen. Nach Holtkamp (2008) ist die Lokalpolitik in kommunalen Konkurrenzdemokratien stärker parteipolitisiert als in Konkordanzdemokratien. Daher sollten in manchen Ländern eher Parteieneffekte feststellbar sein als in anderen. Um dies zu prüfen, wurden die Modelle separat für einzelne Länder berechnet. Die Ergebnisse präsentiert Tab. 4. Aus Gründen der Übersichtlichkeit sind nur die Koeffizienten der Parteivariablen dargestellt. Anhand des Konkordanzindex von Holtkamp lassen sich die Länder beiden Demokratietypen wie folgt zuordnen: Nordrhein-Westfalen und Hessen sind kommunale Konkurrenzdemokratien. Demgegenüber ist die Lokalpolitik in Baden-Württemberg, Rheinland-Pfalz und Thüringen konkordanzdemokratisch geprägt. Bayern hat eine Mittelposition inne.

Auch die länderbezogene Auswertung der Daten zeigt nur vereinzelt signifikante Parteieneffekte, die sich zudem nicht entlang der Dichotomie Konkordanz- und Konkurrenzdemokratie anordnen lassen. Im Fall der Gewerbesteuer kann man für Hessen entgegen den Erwartungen beobachten, dass der Wechsel von parteilosen zu linken Bürgermeistern eine Absenkung des Hebesatzes um vier Punkte bewirkt. In den übrigen Ländern stellen die parteipolitische Ausrichtung des Rates und des Bürgermeisters insignifikante Einflussfaktoren dar, deren Koeffizienten oft unerwartete Vorzeichen aufweisen. Etwas häufiger sind signifikante Parteieneffekte bei den Grundsteuern. Bei der Grundsteuer A bewirkt in Rheinland-Pfalz der Übergang von unklaren Mehrheitsverhältnissen zu einer bürgerlichen Mehrheit eine Verringerung der Hebesätze um drei Punkte, der Wechsel von parteilosen zu linken Bürgermeistern einen Anstieg um zwei Punkte. Entgegen den Erwartungen zeigt sich in Hessen ein negativer Effekt linker Ratsmehrheiten. Auch bei der Grundsteuer B sind signifikante Parteieneffekte festzustellen. Im Einklang mit der Theorie führt in Rheinland- 
Tab. 4 Determinanten der Realsteuerhebesatzpolitik (2004-2013), nach Ländern

\begin{tabular}{|c|c|c|c|c|c|c|}
\hline Sample & NW & $\mathrm{HE}$ & $\mathrm{RP}$ & BW & BY & $\mathrm{TH}$ \\
\hline \multicolumn{7}{|c|}{ Gewerbesteuer (Modell 1a) } \\
\hline $\begin{array}{l}\text { Bürgerliche Mehrheit } \\
\text { im Rat }\end{array}$ & $\begin{array}{c}1,358 \\
(1,082)\end{array}$ & $\begin{array}{c}0,679 \\
(1,375)\end{array}$ & $\begin{array}{r}-0,732 \\
(0,743)\end{array}$ & $\begin{array}{c}1,012 \\
(0,592)\end{array}$ & $\begin{array}{c}0,232 \\
(1,155)\end{array}$ & $\begin{array}{r}-1,575 \\
(2,063)\end{array}$ \\
\hline $\begin{array}{l}\text { Linke Mehrheit im } \\
\text { Rat }\end{array}$ & $\begin{array}{c}-0,026 \\
(2,042)\end{array}$ & $\begin{array}{c}-1,871 \\
(1,196)\end{array}$ & $\begin{array}{c}-0,994 \\
(0,709)\end{array}$ & $\begin{array}{c}-1,105 \\
(1,868)\end{array}$ & $\begin{array}{c}-0,656 \\
(3,218)\end{array}$ & $\begin{array}{l}4,504 \\
(3,558)\end{array}$ \\
\hline $\begin{array}{l}\text { Bürgerlich-konservati- } \\
\text { ver Bürgermeister }\end{array}$ & $\begin{array}{l}-2,060 \\
(2,708)\end{array}$ & $\begin{array}{r}-1,728 \\
(1,700)\end{array}$ & $\begin{array}{l}-0,068 \\
(0,637)\end{array}$ & $\begin{array}{c}0,735 \\
(0,567)\end{array}$ & $\begin{array}{c}0,652 \\
(0,896)\end{array}$ & $\begin{array}{c}-0,656 \\
(2,376)\end{array}$ \\
\hline Linker Bürgermeister & $\begin{array}{c}2,639 \\
(2,799)\end{array}$ & $\begin{array}{r}-3,673^{*} \\
(1,479) \\
\end{array}$ & $\begin{array}{r}-0,263 \\
(0,642) \\
\end{array}$ & $\begin{array}{c}0,052 \\
(0,908) \\
\end{array}$ & $\begin{array}{c}-1,155 \\
(1,140)\end{array}$ & $\begin{array}{c}1,348 \\
(3,444) \\
\end{array}$ \\
\hline \multicolumn{7}{|c|}{ Grundsteuer A (Modell 1b) } \\
\hline $\begin{array}{l}\text { Bürgerliche Mehrheit } \\
\text { im Rat }\end{array}$ & $\begin{array}{c}1,866 \\
(2,162)\end{array}$ & $\begin{array}{c}0,427 \\
(3,204)\end{array}$ & $\begin{array}{r}-2,878 * \\
(1,363)\end{array}$ & $\begin{array}{c}1,251 \\
(1,161)\end{array}$ & $\begin{array}{c}-0,340 \\
(0,719)\end{array}$ & $\begin{array}{c}-2,117 \\
(2,639)\end{array}$ \\
\hline $\begin{array}{l}\text { Linke Mehrheit im } \\
\text { Rat }\end{array}$ & $\begin{array}{c}1,884 \\
(4,086)\end{array}$ & $\begin{array}{r}-5,678^{*} \\
(2,617)\end{array}$ & $\begin{array}{l}-0,719 \\
(1,722)\end{array}$ & $\begin{array}{c}2,835 \\
(5,670)\end{array}$ & $\begin{array}{l}-2,786 \\
(2,836)\end{array}$ & $\begin{array}{c}1,148 \\
(3,786)\end{array}$ \\
\hline $\begin{array}{l}\text { Bürgerlich-konservati- } \\
\text { ver Bürgermeister }\end{array}$ & $\begin{array}{c}3,195 \\
(4,316)\end{array}$ & $\begin{array}{c}-0,331 \\
(4,698)\end{array}$ & $\begin{array}{l}-1,829 \\
(1,285)\end{array}$ & $\begin{array}{c}0,915 \\
(1,219)\end{array}$ & $\begin{array}{l}-0,127 \\
(0,605)\end{array}$ & $\begin{array}{c}0,254 \\
(2,894)\end{array}$ \\
\hline Linker Bürgermeister & $\begin{array}{c}-4,352 \\
(4,628) \\
\end{array}$ & $\begin{array}{c}-2,933 \\
(3,391)\end{array}$ & $\begin{array}{r}2,128 * \\
(1,104)\end{array}$ & $\begin{array}{c}-1,394 \\
(2,069)\end{array}$ & $\begin{array}{r}-1,293 \\
(1,181)\end{array}$ & $\begin{array}{c}-1,569 \\
(4,474)\end{array}$ \\
\hline \multicolumn{7}{|c|}{ Grundsteuer B (Modell 1c) } \\
\hline $\begin{array}{l}\text { Bürgerliche Mehrheit } \\
\text { im Rat }\end{array}$ & $\begin{array}{l}6,643^{*} \\
(2,900)\end{array}$ & $\begin{array}{c}-0,417 \\
(2,289)\end{array}$ & $\begin{array}{r}-2,692 * \\
(1,280)\end{array}$ & $\begin{array}{c}0,753 \\
(1,826)\end{array}$ & $\begin{array}{c}-1,113 \\
(0,693)\end{array}$ & $\begin{array}{l}3,775 \\
(2,239)\end{array}$ \\
\hline $\begin{array}{l}\text { Linke Mehrheit im } \\
\text { Rat }\end{array}$ & $\begin{array}{l}5,515 \\
(4,047)\end{array}$ & $\begin{array}{c}-0,781 \\
(2,444)\end{array}$ & $\begin{array}{l}-0,770 \\
(1,321)\end{array}$ & $\begin{array}{c}8,070 \\
(7,362)\end{array}$ & $\begin{array}{c}0,532 \\
(3,328)\end{array}$ & $\begin{array}{r}8,024^{*} \\
(4,034)\end{array}$ \\
\hline $\begin{array}{l}\text { Bürgerlich-konservati- } \\
\text { ver Bürgermeister }\end{array}$ & $\begin{array}{l}-9,974 \\
(11,878)\end{array}$ & $\begin{array}{r}-1,087 \\
(3,464)\end{array}$ & $\begin{array}{c}1,173 \\
(1,414)\end{array}$ & $\begin{array}{l}-3,104 \\
(2,058)\end{array}$ & $\begin{array}{c}0,152 \\
(0,549)\end{array}$ & $\begin{array}{c}2,211 \\
(2,770)\end{array}$ \\
\hline Linker Bürgermeister & $\begin{array}{l}-0,136 \\
(11,582)\end{array}$ & $\begin{array}{c}-1,696 \\
(2,796)\end{array}$ & $\begin{array}{c}-1,147 \\
(1,190)\end{array}$ & $\begin{array}{l}-4,120 \\
(2,596)\end{array}$ & $\begin{array}{c}0,663 \\
(0,980)\end{array}$ & $\begin{array}{r}-1,412 \\
(3,205)\end{array}$ \\
\hline $\begin{array}{l}\mathrm{R}^{2} \text { (Within), Gewerbe- } \\
\text { steuer (Modell 1a) }\end{array}$ & 0,479 & 0,404 & 0,314 & 0,249 & 0,192 & 0,661 \\
\hline $\begin{array}{l}\mathrm{R}^{2} \text { (Within), Grund- } \\
\text { steuer A (Modell 1b) }\end{array}$ & 0,371 & 0,298 & 0,541 & 0,239 & 0,420 & 0,599 \\
\hline $\begin{array}{l}\mathrm{R}^{2} \text { (Within), Grund- } \\
\text { steuer B (Modell 1c) }\end{array}$ & 0,540 & 0,498 & 0,691 & 0,340 & 0,438 & 0,720 \\
\hline Anzahl Beobachtungen & 3564 & 3833 & 15.043 & 9767 & 18.380 & 6888 \\
\hline $\begin{array}{l}\text { Anzahl Untersu- } \\
\text { chungseinheiten }\end{array}$ & 396 & 426 & 2077 & 1109 & 2054 & 994 \\
\hline
\end{tabular}

Dargestellt sind unstandardisierte Regressionskoeffizienten (panel-robuste Standardfehler in Klammern) Alle Modelle enthalten time-Dummies für einzelne Untersuchungsjahre (Basisjahr: 2005; nicht dargestellt) sowie die weiteren Kontrollvariablen aus dem Grundmodell (Tab. 3)

$* p<0,05$ (zweiseitiger Test)

Pfalz der Wechsel zu bürgerlichen Ratsmehrheiten zu einer Absenkung der Hebesätze um knapp drei Punkte. In Nordrhein-Westfalen zeigt sich überraschend, dass bürgerliche Ratsmehrheiten einen Anstieg des Hebesatzes um sieben Punkte bewirken. Demgegenüber lassen sich in Thüringen beim Übergang zu einer linken Mehrheit wie erwartet steigende Hebesätze beobachten. Alles in allem offenbart die länderbezogene Analyse nur eine begrenzte Parteipolitisierung der Hebesatzpolitik. 
Am ehesten zeigt sich diese bei den Grundsteuern, bei denen nicht nur häufiger Parteieneffekte festzustellen sind, sondern diese auch stärker ausfallen. Dies dürfte daran liegen, dass bei den Grundsteuern aufgrund der immobilen Bemessungsgrundlage der interkommunale Steuerwettbewerb schwächer ausfällt und Parteien mehr Gestaltungsspielraum besitzen.

Neben der Kommunalverfassung wird der Gemeindegröße Einfluss auf die Parteipolitisierung der Lokalpolitik zugeschrieben. Demnach steigt der Grad der Parteipolitisierung mit der Einwohnerzahl an. Erstens ist in größeren Kommunen der Sitzanteil freier Wählervereinigungen, denen eine stärkere Sachorientierung unterstellt wird, geringer. Etablierte Parteien spielen eine wichtigere Rolle. Zweitens zeichnet sich das administrative Führungspersonal häufiger durch eine Parteimitgliedschaft aus, wodurch parteipolitische Interessen auch über die Verwaltungsspitze in den Entscheidungsprozess eingebracht werden. Drittens sind Debatten im Stadtrat häufig allgemeinpolitischer Natur, die Ratsarbeit sowie das Selbstverständnis der Ratsmitglieder stärker parlamentarisch organisiert (Holtmann 1992). Daher sollten Parteieneffekte eher in größeren Städten beobachtbar sein. Um dies zu prüfen, wurde das Sample nach Stadttypen untergliedert und die Modelle gruppenweise geschätzt. In Anlehnung an das Bundesinstitut für Bau-, Stadt- und Raumforschung wurden die Kommunen in fünf Typen klassifiziert: Großstädte (über 100.000 Einwohner), Mittelstädte (20.000-100.000 Einwohner), Größere Kleinstädte (10.000-20.000 Einwohner), Kleinere Kleinstädte (5000-10.000 Einwohner) und Landgemeinden (unter 5000 Einwohner). Die Ergebnisse für die Parteivariablen präsentiert Tab. 5.

Auch die Auswertung nach Stadttypen zeigt nur vereinzelt signifikante Parteieneffekte, am ehesten bei Großstädten und überraschenderweise bei kleineren Kleinstädten, wobei diese bei ersteren deutlich stärker sind. Im Fall der Gewerbesteuer geht in Großstädten wie erwartet der Wechsel von parteilosen zu konservativen Bürgermeistern mit einer Absenkung des Hebesatzes um etwa neun Punkte einher. Wider Erwarten ist auch für linke Ratsmehrheiten ein signifikant negativer Effekt festzustellen. Allerdings ist dieser nur halb so groß. Überdies lässt sich bei kleineren Kleinstädten ein signifikanter Parteieneffekt beobachten. Entgegen den Erwartungen geht ein Übergang von unklaren Mehrheitsverhältnissen zu bürgerlichen Mehrheiten mit einem Anstieg der Hebesätze um zwei Punkte einher. Die übrigen Koeffizienten sind insignifikant und weisen mehrheitlich ein den theoretischen Überlegungen widersprechendes Vorzeichen auf. Bei der Grundsteuer A lässt sich nur in Landgemeinden ein signifikanter Parteieneffekt identifizieren. Im Falle eines Wechsels von unklaren Mehrheitsverhältnissen zu bürgerlichen Ratsmehrheiten sinken die Hebesätze leicht. In allen anderen Fällen sind die Effekte insignifikant und die Koeffizienten weisen häufig ein den theoretischen Annahmen widersprechendes Vorzeichen auf. Dass nur in Landgemeinden ein Parteieneffekt beobachtbar ist, dürfte daran liegen, dass diese oftmals agrarisch geprägt sind und die Grundsteuer A eine wichtigere Rolle spielt. Am ehesten mit den Erwartungen der Parteiendifferenztheorie kompatibel sind die Befunde für die Grundsteuer B. Bezüglich der parteipolitischen Ausrichtung des Rates zeigen sich in Großstädten die erwarteten Effekte: Während der Wechsel zu einer linken Ratsmehrheit einen deutlichen Anstieg der Hebesätze bewirkt, führen bürgerliche Mehrheiten zu einer leichten Absenkung, wobei deren Effekt insignifikant ist. Überraschend ist lediglich, dass Bürgermeister jeglicher 
Tab. 5 Determinanten der Realsteuerhebesatzpolitik (2004-2013), nach Stadttypen

\begin{tabular}{|c|c|c|c|c|c|}
\hline Sample & Großstädte & Mittelstädte & $\begin{array}{l}\text { Größere } \\
\text { Kleinstädte }\end{array}$ & $\begin{array}{l}\text { Kleinere } \\
\text { Kleinstädte }\end{array}$ & $\begin{array}{l}\text { Landge- } \\
\text { meinden }\end{array}$ \\
\hline \multicolumn{6}{|l|}{ Gewerbesteuer (Modell 1a) } \\
\hline $\begin{array}{l}\text { Bürgerliche Mehrheit } \\
\text { im Rat }\end{array}$ & $\begin{array}{c}1,976 \\
(3,091)\end{array}$ & $\begin{array}{c}1,414 \\
(1,153)\end{array}$ & $\begin{array}{l}-0,922 \\
(1,252)\end{array}$ & $\begin{array}{l}2,030 * \\
(0,950)\end{array}$ & $\begin{array}{r}-0,967 \\
(0,892)\end{array}$ \\
\hline Linke Mehrheit im Rat & $\begin{array}{r}-4,201 * \\
(2,122)\end{array}$ & $\begin{array}{c}1,323 \\
(1,710)\end{array}$ & $\begin{array}{l}-0,725 \\
(1,858)\end{array}$ & $\begin{array}{c}0,306 \\
(1,695)\end{array}$ & $\begin{array}{c}0,016 \\
(1,348)\end{array}$ \\
\hline $\begin{array}{l}\text { Bürgerlich-konservati- } \\
\text { ver Bürgermeister }\end{array}$ & $\begin{array}{r}-8,605^{*} \\
(4,051)\end{array}$ & $\begin{array}{c}1,425 \\
(1,462)\end{array}$ & $\begin{array}{c}0,001 \\
(0,979)\end{array}$ & $\begin{array}{c}0,754 \\
(1,007)\end{array}$ & $\begin{array}{r}-0,315 \\
(0,798)\end{array}$ \\
\hline Linker Bürgermeister & $\begin{array}{l}-3,565 \\
(3,233)\end{array}$ & $\begin{array}{l}-0,420 \\
(1,643)\end{array}$ & $\begin{array}{l}-2,338 \\
(1,238)\end{array}$ & $\begin{array}{l}-2,179 \\
(1,182)\end{array}$ & $\begin{array}{r}-0,436 \\
(0,924)\end{array}$ \\
\hline \multicolumn{6}{|l|}{ Grundsteuer A (Modell 1b) } \\
\hline $\begin{array}{l}\text { Bürgerliche Mehrheit } \\
\text { im Rat }\end{array}$ & $\begin{array}{l}0,725 \\
(8,041)\end{array}$ & $\begin{array}{l}3,074 \\
(2,247)\end{array}$ & $\begin{array}{l}-0,562 \\
(2,756)\end{array}$ & $\begin{array}{c}0,427 \\
(1,253)\end{array}$ & $\begin{array}{r}-3,064^{*} \\
(0,959)\end{array}$ \\
\hline Linke Mehrheit im Rat & $\begin{array}{l}-6,846 \\
(5,352)\end{array}$ & $\begin{array}{c}5,991 \\
(4,527)\end{array}$ & $\begin{array}{l}-8,389 \\
(5,358)\end{array}$ & $\begin{array}{l}-2,513 \\
(2,021)\end{array}$ & $\begin{array}{r}-1,538 \\
(1,707)\end{array}$ \\
\hline $\begin{array}{l}\text { Bürgerlich-konservati- } \\
\text { ver Bürgermeister }\end{array}$ & $\begin{array}{l}-5,935 \\
(6,032)\end{array}$ & $\begin{array}{l}-2,563 \\
(4,036)\end{array}$ & $\begin{array}{c}1,319 \\
(3,403)\end{array}$ & $\begin{array}{l}-1,305 \\
(1,028)\end{array}$ & $\begin{array}{c}0,361 \\
(0,701)\end{array}$ \\
\hline Linker Bürgermeister & $\begin{array}{l}-8,501 \\
(5,478)\end{array}$ & $\begin{array}{l}-3,592 \\
(5,790)\end{array}$ & $\begin{array}{l}-2,613 \\
(2,679)\end{array}$ & $\begin{array}{c}-1,719 \\
(1,490)\end{array}$ & $\begin{array}{c}0,223 \\
(1,041)\end{array}$ \\
\hline \multicolumn{6}{|l|}{ Grundsteuer B (Modell 1c) } \\
\hline $\begin{array}{l}\text { Bürgerliche Mehrheit } \\
\text { im Rat }\end{array}$ & $\begin{array}{c}-0,671 \\
(10,669)\end{array}$ & $\begin{array}{c}0,437 \\
(2,179)\end{array}$ & $\begin{array}{c}2,209 \\
(1,938)\end{array}$ & $\begin{array}{c}0,011 \\
(1,362)\end{array}$ & $\begin{array}{r}-1,600 \\
(0,925)\end{array}$ \\
\hline Linke Mehrheit im Rat & $\begin{array}{r}14,780 * \\
(6,977)\end{array}$ & $\begin{array}{c}6,521 \\
(4,847)\end{array}$ & $\begin{array}{r}-1,021 \\
(3,693)\end{array}$ & $\begin{array}{l}4,497 * \\
(2,227)\end{array}$ & $\begin{array}{c}1,244 \\
(1,798)\end{array}$ \\
\hline $\begin{array}{l}\text { Bürgerlich-konservati- } \\
\text { ver Bürgermeister }\end{array}$ & $\begin{array}{l}14,034 \\
(8,446)\end{array}$ & $\begin{array}{c}0,692 \\
(3,054)\end{array}$ & $\begin{array}{c}0,408 \\
(2,399)\end{array}$ & $\begin{array}{r}-5,321 * \\
(1,858)\end{array}$ & $\begin{array}{l}1,618 \\
(0,852)\end{array}$ \\
\hline Linker Bürgermeister & $\begin{array}{l}13,496 \\
(7,426)\end{array}$ & $\begin{array}{l}-0,316 \\
(4,075)\end{array}$ & $\begin{array}{r}-1,564 \\
(2,763)\end{array}$ & $\begin{array}{l}-2,710 \\
(1,443)\end{array}$ & $\begin{array}{r}-0,933 \\
(1,135)\end{array}$ \\
\hline $\begin{array}{l}\mathrm{R}^{2} \text { (Within), Gewerbe- } \\
\text { steuer (Modell 1a) }\end{array}$ & 0,486 & 0,352 & 0,351 & 0,288 & 0,368 \\
\hline $\begin{array}{l}\mathrm{R}^{2} \text { (Within), Grund- } \\
\text { steuer A (Modell 1b) }\end{array}$ & 0,402 & 0,348 & 0,277 & 0,365 & 0,439 \\
\hline $\begin{array}{l}\mathrm{R}^{2} \text { (Within), Grund- } \\
\text { steuer B (Modell 1c) }\end{array}$ & 0,600 & 0,460 & 0,472 & 0,419 & 0,515 \\
\hline Anzahl Beobachtungen & 513 & 3753 & 5160 & 8095 & 39.954 \\
\hline $\begin{array}{l}\text { Anzahl Untersuchungs- } \\
\text { einheiten }\end{array}$ & 59 & 447 & 628 & 994 & 5116 \\
\hline
\end{tabular}

Dargestellt sind unstandardisierte Regressionskoeffizienten (panel-robuste Standardfehler in Klammern) Alle Modelle enthalten time-Dummies für einzelne Untersuchungsjahre (Basisjahr: 2005; nicht dargestellt) sowie die weiteren Kontrollvariablen aus dem Grundmodell (Tab. 3)

$* p<0,05$ (zweiseitiger Test)

Couleur einen positiven Effekt entfalten, der allerdings beide Male insignifikant ist (bei linken Bürgermeistern jedoch nur knapp). Insbesondere der relativ starke, positive Effekt linker Mehrheiten auf die Grundsteuerhebesätze, der sich in Großstädten zeigt, deutet nochmals darauf hin, dass die bloße Möglichkeit der Überwälzung der Grundsteuer auf die Mietkosten linke Parteien nicht davon abzuhalten scheint, an der Steuerschraube zu drehen, denn gerade in den Großstädten dürften die Wohnungs- 
märkte am angespanntesten sein und folglich müsste sich ein etwaiger bremsender Effekt in diesen Kommunen am ehesten beobachten lassen. Auch bei kleineren Kleinstädten sind zwei signifikante, den theoretischen Erwartungen entsprechende Parteieneffekte festzustellen. Der Wechsel zu einer linken Mehrheit geht mit einem Anstieg der Hebesätze um etwa vier Punkte einher, die Ablösung eines parteilosen durch einen konservativen Bürgermeister bewirkt eine Absenkung um fünf Punkte. Im Übrigen sind die Effekte insignifikant, ihre Wirkrichtung widerspricht oftmals den theoretischen Vorüberlegungen.

Sowohl das allgemeine Regressionsmodell als auch die länderweise und stadttypbezogene Auswertung der Daten liefert somit nur begrenzte Evidenz für eine Parteipolitisierung der lokalen Steuerpolitik. Eine mögliche Ursache für das Ausbleiben von Parteieffekten dürfte darin bestehen, dass das aus methodischen Gründen erforderliche Fixed-Effects-Modell lediglich die Varianz innerhalb der Beobachtungseinheiten über die Zeit (within-Varianz) berücksichtigt, nicht jedoch die Varianz zwischen den Untersuchungseinheiten (between-Varianz). Die within-Varianz fällt bei den parteibezogenen Variablen jedoch deutlich niedriger aus, da sowohl die Mehrheitsverhältnisse im Rat wie auch die Bürgermeister nicht im Jahresturnus variieren (wie die anderen unabhängigen Variablen), sondern nur bei Wahlen wechseln können. ${ }^{5}$ Da zudem Bürgermeister häufig wiedergewählt und Ratsmehrheiten verteidigt werden, reduziert sich die within-Varianz noch stärker. Der FixedEffects-Schätzer kann jedoch zur Schätzung des Parteieneffekts nur solche Untersuchungsjahre heranziehen, in denen sich die Mehrheitsverhältnisse im Rat oder die Parteizugehörigkeit des Bürgermeisters verändert haben, was je nach betrachteter Variable in lediglich ein bis zwei Prozent der Beobachtungsjahre der Fall ist. Dies könnte erklären, warum sich nur begrenzt Parteieneffekte identifizieren lassen. Zumindest mit Blick auf die Rolle der im Stadtrat vertretenen Parteien lässt sich das Problem der schmalen Datengrundlage jedoch durch eine alternative Variablenspezifikation etwas abmildern. Verwendet man statt der Mehrheits-Dummies den Sitzanteil der linken und bürgerlichen Parteien im Rat, werden im Gesamtmodell immerhin sechs bis sieben Prozent der Beobachtungsjahre zur Schätzung des Parteieneffekts berücksichtigt (je nach Bundesland und Stadttyp steigt der Anteil sogar auf zehn bis zwanzig Prozent). Dies liegt daran, dass sich die Sitzanteile der Parteien ändern können, ohne dass sich dies in einer Veränderung des MehrheitsDummies niederschlägt. Allerdings lassen sich auch bei einer Neuberechnung der Modelle mit dieser alternativen Variablenspezifikation nur sporadisch signifikante Parteieneffekte feststellen. Im Großen und Ganzen bleiben die zuvor geschilderten Befunde weitgehend erhalten.

Überdies dürften die Möglichkeiten parteigebundener Bürgermeister, die Hebesatzpolitik entsprechend ihrer Präferenzen zu beeinflussen, direkt von den Mehrheitsverhältnissen im Stadtrat abhängen. Parteipolitische Differenzen aufgrund unterschiedlicher Parteizugehörigkeit sind v.a. dann zu erwarten, wenn sich Bürgermeister auf Ratsmehrheiten mit gleichen politischen Überzeugungen stützen können

\footnotetext{
5 Im Untersuchungszeitraum gab es je nach Land ein oder zwei Kommunalwahlen. Da die Amtszeit des Bürgermeisters in den betrachteten Ländern in der Regel 6-8 Jahre beträgt, haben in den meisten Kommunen ebenfalls nur ein oder zwei Bürgermeisterwahlen stattgefunden.
} 
(gleichgerichtete Mehrheit). Im Falle einer Kohabitation, bei der Bürgermeister und Ratsmehrheit unterschiedlichen Lagern angehören, sollten parteigebundene Bürgermeister weniger zur Umsetzung ihrer politischen Präferenzen fähig sein, da sie über keine Hausmacht im Rat verfügen und die Gefahr institutioneller Blockaden infolge einer Obstruktionspolitik durch die oppositionelle Ratsmehrheit steigt (Holtkamp 2002, S. 67; Timm-Arnold 2011, S. 65 ff.). Daher sollten Effekte parteigebundener Bürgermeister eher beobachtbar sein, wenn sie sich auf gleichgerichtete Ratsmehrheiten stützen können. Um dies zu prüfen, wurden die Grundmodelle um Interaktionseffekte zwischen den Mehrheitsverhältnissen im Rat und der Parteifarbe des Bürgermeisters ergänzt. Zur einfacheren Interpretation wurde das Modell ohne die Haupteffekte für die Parteivariablen geschätzt. ${ }^{6}$ Dadurch kann man die Effekte linker bzw. konservativer Bürgermeister (mit/ohne eigene Ratsmehrheit) direkt aus dem Modell ablesen. Die Koeffizienten zeigen den Effekt einzelner Fallkonstellationen im Vergleich zu einer Situation, in der ein parteiloser Bürgermeister amtiert und kein ideologisches Lager die Ratsmehrheit innehat. Tab. 6 präsentiert die Ergebnisse für die Parteivariablen. Bei allen Realsteuern gehen bürgerlich-konservative Verwaltungschefs ohne eigene Ratsmehrheit mit einem leichten Anstieg des Hebesatzes einher, wohingegen sie einen hebesatzbegrenzenden Effekt entfalten, wenn sie sich auf eine eigene Ratsmehrheit stützen. Dieser Zusammenhang ist bei der Grundsteuer A am stärksten ausgeprägt. Demgegenüber weisen linke Amtsinhaber durchgängig einen negativen Effekt auf die Hebesätze auf, unabhängig davon, ob sie sich in einer Kohabitation befinden oder nicht. Nur bei der Grundsteuer B zeigt sich der erwartete Zusammenhang: Sobald sich ein linker Bürgermeister auf eine eigene Ratsmehrheit stützen kann, hat er einen positiven Effekt. Allerdings sind die Parteieneffekte durchgängig insignifikant, insofern dürfen die Ergebnisse nicht überbewertet werden. Neben der Schätzung der Koeffizienten für einzelne Gruppen ist v. a. die Frage interessant, ob sich die Effekte der Gruppen signifikant voneinander unterscheiden. Um dies zu prüfen, wurden F-Tests auf Gruppenunterschiede durchgeführt. Während bei der Gewerbesteuer keine Gruppenunterschiede beobachtbar sind, ist bei den Grundsteuern das Gegenteil der Fall. Der F-Test bei der Grundsteuer A deutet an, dass sich die Effekte konservativer Bürgermeister unterscheiden, je nachdem, ob sie sich auf eine eigene Ratsmehrheit stützen können oder nicht. Der gleiche Befund zeigt sich im Falle der Grundsteuer B für linke Verwaltungschefs.

Nach der Analyse potenzieller Parteieneffekte soll abschließend der fiskalische Problemdruck noch einmal näher betrachtet werden. Wie im Theorieteil aufgezeigt, kann dieser auf zwei Wegen die Hebesatzpolitik beeinflussen: durch die Einsicht lokaler Entscheider in die Notwendigkeit einer Haushaltskonsolidierung oder durch äußeren Druck staatlicher Aufsichtsbehörden. Besonders der Einfluss der Aufsichtsbehörden ist empirisch schwierig zu fassen. Um diesen $\mathrm{zu}$ approximieren, wurde das Grundmodell um eine Dummy-Variable erweitert, die anzeigt, ob Kommunen an einem kommunalen Entschuldungsprogramm teilnahmen. Letztere fanden als Reaktion auf zunehmende lokale Haushaltskrisen in der jüngeren Vergangenheit

\footnotetext{
6 Mathematisch ist das geschätzte Modell äquivalent zu einem Modell mit Haupteffekten. Lediglich die Koeffizienten des Interaktionsterms und deren Interpretation ändern sich durch die (Nicht-)Berücksichtigung der Haupteffekte.
} 
Tab. 6 Determinanten der Realsteuerhebesatzpolitik (2004-2013), Grundmodell mit Interaktion zwischen Ratsmehrheit und Bürgermeister

\begin{tabular}{lccc}
\hline & $\begin{array}{c}\text { Modell 2a } \\
\text { Gewerbesteuer }\end{array}$ & $\begin{array}{c}\text { Modell 2b } \\
\text { Grundsteuer A }\end{array}$ & $\begin{array}{c}\text { Modell 2c } \\
\text { Grundsteuer B }\end{array}$ \\
\hline $\begin{array}{l}\text { Interaktionseffekte } \\
\text { Bürgerlich-konservativer Bürger- }\end{array}$ & 0,220 & & \\
$\begin{array}{l}\text { meister } \\
\text { (ohne eigene Ratsmehrheit) }\end{array}$ & $(0,594)$ & $(0,104$ & 0,032 \\
Bürgerliche Mehrheit & & & $(0,762)$ \\
(ohne eigenen Bürgermeister) & 0,656 & $-1,448$ & 0,728 \\
$\begin{array}{l}\text { Bürgerlich-konservativer Bürger- } \\
\text { meister }\end{array}$ & $(0,746)$ & $(1,121)$ & $(1,320)$ \\
(mit eigener Ratsmehrheit) & $-0,197$ & $-1,629$ & $-1,198$ \\
$\begin{array}{l}\text { Linker Bürgermeister } \\
\text { (ohne eigene Ratsmehrheit) }\end{array}$ & $(0,837)$ & $(1,058)$ & $(1,060)$ \\
$\begin{array}{l}\text { Linke Mehrheit } \\
\text { (ohne eigenen Bürgermeister) }\end{array}$ & $-0,932$ & $-1,094$ & $-1,034$ \\
$\begin{array}{l}\text { Linker Bürgermeister } \\
\text { (mit eigener Ratsmehrheit) }\end{array}$ & $(0,645)$ & $(0,840)$ & $(0,853)$ \\
\hline
\end{tabular}

Test auf signifikanten Unterschied zwischen bürgerlich-konservativem Bürgermeister mit/ohne eigene Mehrheit im Rat
F-Test ( $p$-Wert)
0,536
0,043
0,135

Test auf signifikanten Unterschied zwischen linkem Bürgermeister mit/ohne eigene Mehrheit im Rat

\begin{tabular}{lccc} 
F-Test $(p$-Wert) & 0,471 & 0,445 & 0,024 \\
\hline $\mathrm{R}^{2}$ (Within) & 0,350 & 0,387 & 0,478 \\
Anzahl Beobachtungen & 57.475 & 57.475 & 57.475 \\
Anzahl Untersuchungseinheiten & 7056 & 7056 & 7056
\end{tabular}

Dargestellt sind unstandardisierte Regressionskoeffizienten (panel-robuste Standardfehler in Klammern) Alle Modelle enthalten time-Dummies für einzelne Untersuchungsjahre (Basisjahr: 2005; nicht dargestellt) sowie die weiteren Kontrollvariablen aus dem Grundmodell (Tab. 3)

$* p<0,05$ (zweiseitiger Test)

massive Verbreitung (Person und Geißler 2020). Im Zuge der Programme wurden die Teilnehmerkommunen einer strikteren Haushaltsaufsicht unterworfen und bei der Erstellung von Sanierungskonzepten eng durch die Aufsichtsbehörden begleitet (Person und Zabler 2017). Dabei forderten diese vehement auch einnahmeseitige Konsolidierungsmaßnahmen. Insofern sollte die Teilnahme an Entschuldungsprogrammen einen positiven Effekt auf die Realsteuerhebesätze entfalten.

Tab. 7 präsentiert die Ergebnisse des erweiterten Modells, nach Ländern aufgeschlüsselt. Dabei ist zu beachten, dass Baden-Württemberg und Thüringen kein Entschuldungsprogramm auflegten. Die Analyse des Gesamtsamples zeigt, dass die Programmteilnahme einen spürbaren Anstieg der Hebesätze bewirkt, zumindest bei den Grundsteuern. Dabei ist der Effekt bei der Grundsteuer B doppelt so hoch wie bei der Grundsteuer A. Dies liegt vermutlich daran, dass deren Bemessungsgrundlage (und damit das Konsolidierungspotential) vielerorts deutlich größer ausfällt. Demgegenüber ist für die Gewerbesteuer ein negativer Effekt festzustellen, der darauf zurückzuführen sein dürfte, dass sich Kommunen bei der Gewerbesteuer einem intensiveren Steuerwettbewerb ausgesetzt sehen und daher zurückhaltender sind, diese 
Tab. 7 Determinanten der Realsteuerhebesatzpolitik (2004-2013), nach Ländern

\begin{tabular}{|c|c|c|c|c|c|c|c|}
\hline Sample & Gesamt & NW & $\mathrm{HE}$ & $\mathrm{RP}$ & BW & $\mathrm{BY}$ & $\mathrm{TH}$ \\
\hline \multicolumn{8}{|c|}{ Gewerbesteuer (Modell 3a) } \\
\hline $\begin{array}{l}\text { Teilnahme Entschul- } \\
\text { dungsprogramm }\end{array}$ & $\begin{array}{r}-1,957 * \\
(0,515)\end{array}$ & $\begin{array}{l}4,456 * \\
(2,218)\end{array}$ & $\begin{array}{c}6,267 * \\
(2,163)\end{array}$ & $\begin{array}{l}1,622 * \\
(0,518)\end{array}$ & $\mathrm{X}$ & $\begin{array}{r}-1,745 \\
(1,311)\end{array}$ & $\mathrm{X}$ \\
\hline \multicolumn{8}{|c|}{ Grundsteuer A (Modell 3b) } \\
\hline $\begin{array}{l}\text { Teilnahme Entschul- } \\
\text { dungsprogramm }\end{array}$ & $\begin{array}{r}11,247 * \\
(1,137)\end{array}$ & $\begin{array}{c}26,235^{*} \\
(7,179)\end{array}$ & $\begin{array}{c}22,379 * \\
(4,704)\end{array}$ & $\begin{array}{l}7,127 * \\
(0,946)\end{array}$ & $\mathrm{X}$ & $\begin{array}{c}1,225 \\
(0,790)\end{array}$ & $\mathrm{X}$ \\
\hline \multicolumn{8}{|c|}{ Grundsteuer B (Modell 3c) } \\
\hline $\begin{array}{l}\text { Teilnahme Entschul- } \\
\text { dungsprogramm }\end{array}$ & $\begin{array}{r}23,452 * \\
(1,171)\end{array}$ & $\begin{array}{r}40,994^{*} \\
(8,602)\end{array}$ & $\begin{array}{r}27,960 * \\
(4,307)\end{array}$ & $\begin{array}{r}16,034 * \\
(0,983)\end{array}$ & $\mathrm{X}$ & $\begin{array}{r}2,997 * \\
(0,814)\end{array}$ & $\mathrm{X}$ \\
\hline $\begin{array}{l}R^{2} \text { (Within), Gewerbe- } \\
\text { steuer (Modell 3a) }\end{array}$ & 0,350 & 0,482 & 0,409 & 0,316 & 0,249 & 0,192 & $\overline{0,661}$ \\
\hline $\begin{array}{l}R^{2} \text { (Within), Grund- } \\
\text { steuer A (Modell 3b) }\end{array}$ & 0,395 & 0,398 & 0,315 & 0,550 & 0,239 & 0,420 & 0,599 \\
\hline $\begin{array}{l}R^{2} \text { (Within), Grund- } \\
\text { steuer B (Modell 3c) }\end{array}$ & 0,501 & 0,570 & 0,521 & 0,720 & 0,340 & 0,438 & 0,720 \\
\hline Anzahl Beobachtungen & 57.475 & 3564 & 3833 & 15.043 & 9767 & 18.380 & 6888 \\
\hline $\begin{array}{l}\text { Anzahl Untersu- } \\
\text { chungseinheiten }\end{array}$ & 7056 & 396 & 426 & 2077 & 1109 & 2054 & 994 \\
\hline
\end{tabular}

Dargestellt sind unstandardisierte Regressionskoeffizienten (panel-robuste Standardfehler in Klammern) Alle Modelle enthalten time-Dummies für einzelne Untersuchungsjahre (Basisjahr: 2005; nicht dargestellt) sowie die weiteren Kontrollvariablen aus dem Grundmodell (Tab. 3)

$* p<0,05$ (zweiseitiger Test)

$\mathrm{X}$ : Kategorie nicht besetzt

zum Zwecke der Haushaltskonsolidierung zu erhöhen. Vielmehr versuchen finanziell angeschlagene Kommunen, eine Erhöhung der Gewerbesteuer so weit möglich zu vermeiden, um ihre Wettbewerbsfähigkeit nicht weiter zu verschlechtern. Sie greifen erst dann darauf zu, wenn ihnen keine andere Wahl bleibt.

Die länderweise Auswertung verdeutlicht, dass der Effekt der Entschuldungsprogramme in Nordrhein-Westfalen am stärksten ist, gefolgt von Hessen und Rheinland-Pfalz. Dies überrascht kaum, da der Konsolidierungsdruck in diesem Land am höchsten sein dürfte. Schließlich plagen sich viele nordrhein-westfälische Kommunen seit etlichen Jahren mit gravierenden Haushaltsproblemen. Angesichts des hohen Konsolidierungsdrucks, dem die Kommunen aller drei Länder ausgesetzt sind, verwundert es nicht, dass alle Realsteuern zur Haushaltskonsolidierung herangezogen werden, auch die Gewerbesteuer, trotz der damit verbundenen Schwächung der eigenen Wettbewerbsfähigkeit. Überraschend ist allenfalls, dass die Effekte in Rheinland-Pfalz wesentlich schwächer ausfallen, schließlich gehört auch RheinlandPfalz seit Jahren mit Blick auf die kommunale Verschuldung zu den Sorgenkindern der Republik. Ein vollkommen anderes Bild zeigt sich für Bayern, wo die Teilnahme am Entschuldungsprogramm nur einen leichten Effekt auf die Hebesätze bei der Grundsteuer B entfaltet. Weder bei der Grundsteuer A noch bei der Gewerbesteuer sind signifikante Effekte festzustellen. Dies dürfte darauf zurückzuführen sein, dass das bayerische Programm hochselektiv war. Es erfasste nur 30 der 2056 Gemeinden des Freistaates und war primär als Pilotprojekt zur Fortentwicklung des kommunalen Finanzausgleichs konzipiert (Person und Geißler 2020). Zusammen- 
fassend bleibt festzuhalten, dass sich der im Rahmen der Entschuldungsprogramme intensivierte Druck staatlicher Aufsichtsbehörden in einem spürbaren Anstieg der Realsteuerhebesätze manifestiert und die Aufsichtsbehörden somit einen relevanten Einflussfaktor der kommunalen Steuerpolitik darstellen.

\section{Fazit}

Die autonome Festlegung der Realsteuerhebesätze stellt ein wichtiges Element der kommunalen Finanzautonomie dar. Da die Kommunen jedoch von ihrem Hebesatzrecht sehr unterschiedlich Gebrauch machen, stellt sich die Frage nach den Determinanten ihrer Hebesatzpolitik. Werden Hebesatzentscheidungen primär durch sozioökonomische Rahmenbedingungen beeinflusst? Sind fiskalischer Problemdruck, Haushaltsnotlagen und explodierende Schulden die wesentlichen Treiber? Und welche Rolle spielen politische Faktoren? Zur Beantwortung dieser Fragen analysierte der Beitrag die Realsteuerhebesatzpolitik der Kommunen in sechs Bundesländern zwischen 2004 und 2013. Dabei wurden nicht nur die bisher in der Literatur zur lokalen Steuerpolitik dominierenden ökonomischen Erklärungsansätze um eine politikwissenschaftliche Perspektive ergänzt, sondern auch der einseitige Fokus auf die Gewerbesteuer durch die Berücksichtigung der Grundsteuern überwunden. Durch die Verwendung aktuellerer Untersuchungsjahre kann zudem geprüft werden, wie sich kommunale Steuerpolitik unter veränderten Rahmenbedingungen infolge von Kommunalverfassungsreformen und zunehmender Haushaltskrisen entfaltet. Überdies erlaubt die Analyse mehrerer Länder, unterschiedliche Gemeindestrukturen und institutionelle Grundlagen der Lokalpolitik zu berücksichtigen. Dies erhöht die Generalisierbarkeit der Befunde.

Im Rahmen der Analyse stellen sich v. a. ökonomische und budgetäre Variablen als erklärungskräftig dar. Insbesondere fiskalischer Problemdruck hat einen starken Einfluss auf die Hebesatzpolitik. Einerseits schlagen sich steigende Schulden in höheren Steuersätzen nieder. Andererseits führt die Teilnahme an kommunalen Entschuldungsprogrammen zu einem spürbaren Anstieg der Hebesätze. Dies ist vorwiegend auf den Druck der Aufsichtsbehörden und deren Forderungen nach einnahmeseitigen Konsolidierungsmaßnahmen zurückzuführen, der sich bei den Programmkommunen intensiviert. Wenig überraschend sind die Effekte bei der Grundsteuer B am stärksten ausgeprägt, die sich sowohl mit Blick auf die Breite als auch die Immobilität der Bemessungsgrundlage besonders als Konsolidierungsinstrument eignet.

Im Hinblick auf das Hebesatzanspannungspotential stellen auch die Arbeitslosenquote und die Gemeindegröße wichtige Determinanten der Hebesatzpolitik dar. In beiden Fällen führt ein Anstieg der Variablen zu einer Reduktion der Hebesätze. Im ersten Fall spiegelt sich darin der Versuch, die eigene Wettbewerbsposition zu verbessern. Im zweiten Fall scheint es, dass wachsende (und prosperierende) Kommunen ihre Bürger und Unternehmen an dieser positiven Entwicklung partizipieren lassen. Mit Blick auf den Hebesatzanspannungsbedarf sind die Finanzkraft und die Bevölkerungsstruktur erklärungskräftige Faktoren. Während eine gute Finanzausstattung die Notwendigkeit zur Anspannung der Hebesätze verringert, führt ein An- 
stieg der Kinderquote, z. T. auch der Seniorenquote, zu höheren Hebesätzen, da die Zahl der besonders ausgabenintensiven Bedarfsträger zunimmt. Lediglich beim Anteil der Personen zwischen 15 und 25 Jahren bestätigt sich der Trend nicht. Überdies zeigt die Analyse, dass die Realsteuerhebesätze nicht als strategische Substitute verwendet werden, sondern sich gleichläufig entwickeln: Eine Hebesatzerhöhung bei einer Realsteuer geht mit Anstiegen bei den anderen einher.

Im Gegensatz dazu sind nur vereinzelt signifikante Parteieneffekte beobachtbar, selbst wenn man nach Bundesländern oder Stadttypen differenziert. Dies stützt die Vermutung, dass der Handlungsspielraum der Parteien auf lokaler Ebene angesichts persistenter Haushaltsprobleme und verstärkter Interventionen der Aufsichtsbehörden sinkt. Am ehesten sind Parteieneffekte in Großstädten und bei den Grundsteuern (v.a. Grundsteuer B) festzustellen. Letzteres dürfte darauf zurückzuführen sein, dass bei den Grundsteuern der Gestaltungsspielraum der Parteien aufgrund der immobilen Bemessungsgrundlage nicht auch noch durch den interkommunalen Steuerwettbewerb unterminiert wird. Schließlich scheint auch das Zusammenspiel zwischen Ratsmehrheit und Bürgermeister für die Hebesatzpolitik von Bedeutung zu sein. Zumindest bei den Grundsteuern gibt es Indizien, dass parteigebundene Bürgermeister v. a. dann ihre steuerpolitischen Präferenzen umsetzen können, wenn sie sich auf eine Ratsmehrheit mit gleicher ideologischer Ausrichtung stützen.

Allerdings unterliegt die im Rahmen der Studie vorgelegte Analyse der Parteieneffekte gewissen Limitationen. Der aus methodischen Gründen notwendige Fixed-Effects-Schätzer kann nur die Varianz innerhalb der Kommunen berücksichtigen. Diese fällt jedoch wie gezeigt relativ gering aus, da sich die parteipolitischen Prädiktoren im Gegensatz zu den anderen unabhängigen Variablen nicht jährlich ändern, sondern allenfalls bei Wahlen. Dadurch erfolgt die Ermittlung des Parteieneffekts nur auf Basis jener Untersuchungsjahre, in denen sich die Mehrheitsverhältnisse im Rat oder die Parteizugehörigkeit des Bürgermeisters verändert. Die übrigen Beobachtungsjahre finden keine Berücksichtigung. Um dieses Problem zu adressieren, könnten zukünftige Studien alternative methodische Verfahren zur Erfassung der Parteieneffekte nutzen. Eine Möglichkeit bestünde darin, die Analyse nicht auf Basis einzelner Haushaltsjahre durchzuführen, sondern ganze Wahl- und Legislaturperioden zugrunde $\mathrm{zu}$ legen (wie dies mittlerweile vereinzelt für die international vergleichende Staatstätigkeitsforschung vorgeschlagen wurde, siehe z.B. Garritzmann und Seng 2016). Da eine Legislaturperiode üblicherweise mehrere Haushaltsjahre umfasst, würden dadurch die Informationen mehrerer Beobachtungsjahre berücksichtigt und die Datenbasis zur Schätzung des Parteieneffektes verbreitert. Eine zweite Möglichkeit bestünde in der Verwendung alternativer ökonometrischer Schätzverfahren, die eine quasi-experimentelle Situation ausnutzen, um den kausalen Effekt politischer Parteien auf die lokale Steuerpolitik identifizieren zu können.

Obwohl das Erklärungsmodell zahlreiche Determinanten der Realsteuerpolitik identifiziert und miteinbezieht, sind zudem weitere Einflussfaktoren denkbar, denen zukünftig verstärkte Beachtung geschenkt werden könnte. Interessant wäre beispielsweise eine Verknüpfung des vorgelegten Ansatzes mit Theorien des Steuerwettbewerbs, indem eine direkte Modellierung des interkommunalen Steuerwettbewerbs im Rahmen räumlicher Regressionsmodelle erfolgt. Dadurch ließen sich Interdependenzen zwischen Kommunen bei ihrer Hebesatzentscheidung berücksichtigen. 
Auch eine Modellierung der Wechselwirkungen zwischen lokaler Hebesatzpolitik und kommunalem Finanzausgleich könnte spannende Erkenntnisse generieren, das Erklärungsmodell verfeinern und zu einem besseren Verständnis der lokalen Haushaltspolitik beitragen. Entsprechende Erweiterungen müssen allerdings zukünftigen Forschungsarbeiten vorbehalten bleiben.

Danksagung Für hilfreiche Anmerkungen zu einer früheren Version dieses Artikels danke ich Steffen Zabler und zwei anonymen GutachterInnen. Alle verbliebenen Fehler sind meine eigenen.

Funding Open Access funding enabled and organized by Projekt DEAL.

Open Access Dieser Artikel wird unter der Creative Commons Namensnennung 4.0 International Lizenz veröffentlicht, welche die Nutzung, Vervielfältigung, Bearbeitung, Verbreitung und Wiedergabe in jeglichem Medium und Format erlaubt, sofern Sie den/die ursprünglichen Autor(en) und die Quelle ordnungsgemäß nennen, einen Link zur Creative Commons Lizenz beifügen und angeben, ob Änderungen vorgenommen wurden.

Die in diesem Artikel enthaltenen Bilder und sonstiges Drittmaterial unterliegen ebenfalls der genannten Creative Commons Lizenz, sofern sich aus der Abbildungslegende nichts anderes ergibt. Sofern das betreffende Material nicht unter der genannten Creative Commons Lizenz steht und die betreffende Handlung nicht nach gesetzlichen Vorschriften erlaubt ist, ist für die oben aufgeführten Weiterverwendungen des Materials die Einwilligung des jeweiligen Rechteinhabers einzuholen.

Weitere Details zur Lizenz entnehmen Sie bitte der Lizenzinformation auf http://creativecommons.org/ licenses/by/4.0/deed.de.

\section{Literatur}

Alesina, Alberto, und Roberto Perotti. 1995. The political economy of budget deficits. IMF Staff Papers 42:1-31.

Beck, Kilian. 2019. Hebesatzpolitik und Beitragsplanung - Empirische Befunde zu den Steuern und Beiträgen auf lokaler Ebene. Würzburg: Würzburg University Press.

Berlemann, Michael, und Jane Tilgner. 2007. Determinanten der innerdeutschen Standortwahl von Unternehmen - Ergebnisse einer empirischen Analyse. Ifo Dresden berichtet 2007:14-22.

Boettcher, Florian. 2013a. Determinanten der kommunalen Hebesatzpolitik (Teil 1). Zeitschrift für Kommunalfinanzen 63:103-108.

Boettcher, Florian. 2013b. Determinanten der kommunalen Hebesatzpolitik (Teil 2). Zeitschrift für Kommunalfinanzen 63:126-129.

Bogumil, Jörg, und Lars Holtkamp. 2006. Kommunalpolitik und Kommunalverwaltung. Eine policyorientierte Einführung. Wiesbaden: VS.

Boix, Carles. 1998. Political parties, growth and equality. Cambridge: Cambridge University Press.

Bucovetsky, Sam. 1991. Assymetric tax competition. Journal of Urban Economics 30:167-181.

Büttner, Thiess. 1999. Nationaler und regionaler Steuerwettbewerb - Problematik und empirische Relevanz. In Fiskalischer Föderalismus in Europa, Hrsg. Herbert Wilkens, 111-142. Berlin: Duncker \& Humblot.

Büttner, Thiess. 2000. Steuerwettbewerb im Föderalstaat: Eine empirische Analyse der kommunalen Hebesatzpolitik. In Finanzverfassung und Föderalismus in Deutschland und Europa, Hrsg. Thiess Büttner, 61-88. Baden-Baden: Nomos.

Büttner, Thiess. 2001. Local business taxation and competition for capital: the choice of the tax rate. Regional Science and Urban Economics 31:215-245.

Büttner, Thiess, und Wolfgang Franz. 2003. Kommunaler Steuerwettbewerb und Arbeitslosigkeit: Eine ökonometrische Analyse. In Instrumente der Finanzpolitik, Hrsg. Klaus G. Adam, Wolfgang Franz, 14-20. Frankfurt: FAZ-Institut für Management, Markt- und Medieninformationen GmbH.

Destatis. Statistik über den Realsteuervergleich. https://www.destatis.de/DE/Themen/Staat/Steuern/ Steuereinnahmen/Tabellen/realsteuervergleich-zeitreihe-realsteuern.html. Zugegriffen: 6. Okt. 2020.

Eckey, Hans-Friedrich. 2008. Regionalökonomie. Wiesbaden: Gabler. 
Fehrenz, Sabrina. 2019. Wer steuert die Kommunalsteuern? In Jahrbuch für öffentliche Finanzen 2019, Hrsg. Martin Junkernheinrich, et al., 527-547. Berlin: BWV.

Fuest, Clemens, und Regina T. Riphahn. 2001. Is the local business tax a user tax? An empirical investigation for Germany. Jahrbücher für Nationalökonomie und Statistik 221:14-31.

Gabriel, Oscar W., Volker Kunz und Thomas Zapf-Schramm. 1994. Parteiideologien und Problemverarbeitung in der kommunalen Infrastrukturpolitik. In Kommunalwissenschaftliche Analysen, Hrsg. Oscar W. Gabriel und Rüdiger Voigt, 139-166. Bochum: Universitätsverlag Dr. N. Brockmeyer.

Garrett, Geoffrey. 1998. Partisan politics in the global economy. Cambridge: Cambridge University Press.

Garritzmann, Julian, und Killian Seng. 2016. Party politics and education spending: challenging some common wisdom. Journal of European Public Policy 23:510-530.

Grabow, Busso, und Beate Hollbach-Grömig. 1994. Zur Bedeutung des Standortfaktors „,Kommunale Steuern und Abgaben“. Der Gemeindehaushalt 95:145-149.

Hallerberg, Mark, und Scott Basinger. 1998. Internationalization and changes in tax policy in OECD countries. Comparative Political Studies 31:321-352.

Hauptmeier, Sebastian, Ferdinand Mittermaier, und Johannes Rincke. 2012. Fiscal competition over taxes and public inputs. Regional Science and Urban Economics 42:407-419.

Hibbs, Douglas. 1977. Political parties and macroeconomic policy. American Political Science Review 71:1467-1487.

Holtkamp, Lars. 2000. Kommunale Haushaltspolitik in NRW. Wiesbaden: VS.

Holtkamp, Lars. 2002. Kommunale Haushaltspolitik in den 90erJahren - Der Wandel von polity, politics und policy. In Kommunale Entscheidungsprozesse im Wandel, Hrsg. Jörg Bogumil, 55-73. Opladen: Leske + Budrich.

Holtkamp, Lars. 2008. Kommunale Konkordanz- und Konkurrenzdemokratie. Wiesbaden: VS.

Holtmann, Everhard. 1992. Politisierung der Kommunalpolitik und Wandlungen im lokalen Parteiensystem. Aus Politik und Zeitgeschichte 42:13-22.

Koh, Hyun-Ju, Nadine Riedel, und Tobias Böhm. 2013. Do governments tax agglomeration rents? Journal of Urban Economics 75:92-106.

Korpi, Walter. 1983. The democratic class struggle. London: Routledge \& Kegan Paul.

Krugman, Paul. 1991. Geography and trade. Cambridge: MIT Press.

Von Kulessa, Alexander, und Georg Wenzelburger. 2015. Starker Steuerwettbewerb - starke Reformen? Ein neuer Blick auf Unternehmenssteuerreformen in 15 EU-Staaten (1998-2011). Swiss Political Science Review 21:302-332.

Kunz, Volker. 1998. Die Hebesatzpolitik der kreisfreien Städte in den 80er Jahren. In Kommunalfinanzen im Umbruch, Hrsg. Heinrich Mäding, Rüdiger Voigt, 161-184. Opladen: Leske + Budrich.

Kunz, Volker. 2000. Parteien und kommunale Haushaltspolitik im Städtevergleich. Wiesbaden: VS.

Olson, Mancur. 1982. The rise and decline of nations. Economic growth, stagflation, and social rigidities. New Haven: Yale University Press.

Person, Christian. 2020. Lokale Steuerpolitik im Spannungsfeld zwischen parteipolitischer Einflussnahme und sozioökonomischem Problemdruck: eine Analyse der Determinanten kommunaler Gewerbesteuerhebesätze am Beispiel Nordrhein-Westfalens. Politische Vierteljahresschrift 61:61-86.

Person, Christian, und René Geißler. 2020. Ein Fass ohne Boden? Vier Jahrzehnte kommunaler Schuldenhilfen in Deutschland. dms - der moderne staat 13:191-216.

Person, Christian, und Steffen Zabler. 2017. Veränderter Rahmen - veränderte Praxis? Auswirkungen kommunaler Entschuldungsfonds auf die Praxis der Finanzaufsicht. Zeitschrift für Kommunalfinanzen 67:1-6.

Postlep, Rolf-Dieter. 1985. Wirtschaftsstruktur und großstädtische Finanzen. Hannover: Vincentz.

Rose, Richard, und Phillip L. Davies. 1994. Inheritance in Public Policy. Change without choice in Britain. New Haven: Yale University Press.

Rose, Richard, und Terence Karran. 1987. Taxation by political inertia. Financing the growth of government in Britain. London: Allen \& Unwin.

Schwarting, Gunnar. 2011. Haushaltskonsolidierung in Kommunen. Leitfaden für Rat und Verwaltung. Berlin: ESV.

Schwarting, Gunnar. 2019. Der kommunale Haushalt. Berlin: ESV.

Seitz, Helmut. 1995. The productivity and supply of urban infrastructures. The Annals of Regional Science 29:121-141.

Timm-Arnold, Klaus-Peter. 2011. Bürgermeister und Parteien in der kommunalen Haushaltspolitik. Wiesbaden: VS.

Tsebelis, George. 2002. Veto Players. How political institutions work. New York: SAGE.

Tufte, Edward R. 1978. Political control of the economy, 2. Aufl., Princeton: Princeton University Press. 
Wagschal, Uwe. 2005. Steuerpolitik und Steuerreformen im internationalen Vergleich. Münster: LIT.

Wagschal, Uwe. 2006. Die Politische Ökonomie der Besteuerung. In Politische Ökonomie. Demokratie und wirtschaftliche Leistungsfähigkeit, Hrsg. Herbert Obinger, Uwe Wagschal, und Bernhard Kittel, 259-288. Wiesbaden: VS.

Wagschal, Uwe. 2015. Öffentliche Finanzen. In Handbuch Policy-Forschung, Hrsg. Georg Wenzelburger, Reimut Zohlnhöfer, 505-533. Wiesbaden: Springer VS.

Wagschal, Uwe, Janine von Wolfersdorff, und Kathrin Andrae. 2016. Update Gewerbesteuer und Grundsteuer. Steuerentwicklung, Steuerwettbewerb und Reformblockaden. Berlin: Institut Finanzen und Steuern e. V.

Wilensky, Harold L. 1975. The welfare state and equality: structural and ideological roots of public expenditure, 4. Aufl., Berkeley: University of California Press.

Wilson, John D. 1991. Tax competition with interregional differences in factor endowments. Regional Science and Urban Economics 21:423-451. 